\title{
Espèces introduites ou invasives des ports du Havre, d'Antifer et de Rouen (Normandie, France)
}

\author{
Alien or invasive species in the ports of Le Havre, Antifer and \\ Rouen (Normandy, France)
}

\section{G. Breton}

\author{
Association Port Vivant, 6 rue des Réservoirs, 76600 Le Havre, France \\ gerardbreton@free.fr
}

\begin{abstract}
Résumé - L'exploration des bassins des ports du Havre, d'Antifer et de Rouen (Normandie, France) en plongée subaquatique en scaphandre autonome, en particulier dans le cadre du programme de recherches « V.I.P. - Vie Introduite dans les Ports » du GIP Seine Aval (20102012) a conduit à un inventaire de 364 espèces animales, dont 36 introduites pour le port du Havre, exploré depuis 1978, 268 espèces dont 13 introduites pour le port d'Antifer et 98 espèces végétales et animales dont 12 introduites, pour le port de Rouen. Les introductions anciennes sont distinguées des introductions récentes. Le caractère invasif ou non de chaque espèce et la dynamique de l'invasion sont discutés. Contrairement aux ports d'Antifer et de Rouen dont les peuplements biologiques sont respectivement identiques à ceux du littoral ou du réseau hydrographique voisins, le port du Havre joue un rôle de site pionnier pour l'accueil d'espèces immigrantes, rôle favorisé par le caractère paralique de ce plan d'eau.
\end{abstract}

Mots clés - espèce introduite, espèce invasive, domaine paralique, Manche orientale, Seine

\begin{abstract}
The exploration of the ports of Le Havre, Antifer and Rouen (Normandy, France) by scuba diving, especially in the framework of the research program "V.I.P. - Vie Introduite dans les Ports [=Introduced Life in Harbours]" of the GIP Seine-Aval (2010-2012), has led to a survey of 364 animal species, of which 36 are non native to the port of Le Havre ( studied since 1978); 268 species of which 13 are non native to the port of Antifer, and 98 animal and vegetal species, of which 12 are non native to the port of Rouen. Ancient and recent introductions are distinguished. The invasive character - or not - of every alien species, as well as the dynamics of the invasion, are discussed. Unlike Antifer and Rouen harbours, where the biological populations are respectively similar to those of the neighbouring coast or hydrographical system, the port of Le Havre plays the role of pioneer site for the settlement of many alien species. This role is favoured by the paralic character of this port.
\end{abstract}

Key words - alien species, invasive species, paralic domain, Eastern Channel, Seine River 


\section{INTRODUCTION}

Après un recensement sommaire initial (Breton, 1981b) et un inventaire partiel (Breton et al., 2002), une synthèse représentant une vingtaine d'années d'observations (Breton, 2005) a dressé une liste de 54 taxons procaryotes et végétaux et 308 taxons animaux reconnus en plongée subaquatique en scaphandre autonome dans les bassins du port du Havre. Ce dernier travail montre que les caractères des peuplements $d u$ port $d u$ Havre sont ceux du domaine paralique ${ }^{1}$ (sensu Guélorget \& Perthuisot, 1983) et une zonation est proposée. Le cadre physique (niveau de l'eau, hydrodynamisme, salinité, température, luminosité, turbidité, teneur en oxygène, confinement, orientation et nature des substrats), décrit en détail (Breton, 2005, pp. 385-391), ne sera pas repris ici. Un chapitre particulier est consacré aux espèces allochtones et aux espèces invasives. Une liste détaillée et annotée des taxons observés est fournie.

Depuis 2005, l'exploration en plongée subaquatique s'est poursuivie dans le cadre de l'activité de l'association Port Vivant. En sept ans, 59 espèces animales non encore recensées ont été reconnues. Une étude, menée en 2010-2011 dans le cadre du programme « VIP - Vie Introduite dans les Ports » du GIP Seine-Aval a élargi les

(1) Le domaine paralique est constitué par les plans d'eau littoraux de transition entre le domaine marin franc (thalassique) et le domaine continental. Les peuplements se répartissent selon une zonation dépendant de l'influence maritime exprimée en chiffres romains de I à VI $(I=$ le plus proche du thalassique à $\mathrm{VI}=$ le paralique le plus lointain). recherches aux ports d'Antifer et de Rouen, ce dernier étant en eau douce. Les recherches se sont plus particulièrement focalisées sur les espèces allochtones (= introduites), et, lorsqu'elles s'avéraient invasives, sur les caractères de l'invasion.

L'objectif de cet article est de dresser une liste des espèces animales introduites recensées dans les ports du Havre, d'Antifer et de Rouen, et de commenter sur la date, le processus d'introduction et les modalités de l'invasion éventuelle. Les taxons végétaux des ports du Havre et d'Antifer feront l'objet d'une publication séparée (Verlaque \& Breton, recherches en cours). On comparera enfin le statut des espèces allochtones des ports de Rouen, d'Antifer et du Havre et les modalités des introductions d'espèces dans ces deux ports.

La liste des espèces recensées dans le port du Havre (Breton, 2005) fait l'objet d'une actualisation nomenclaturale (annexe 1) et systématique (taxons douteux, modifications d'identifications) (annexe 2). La liste des taxons animaux nouvellement recensés depuis 2005 est donnée en annexe 3. Une liste complète des taxons animaux du port d'Antifer, et une liste des animaux et végétaux du port de Rouen sont données respectivement en annexes 4 et 5 .

\section{MATÉRIEL ET MÉTHODES}

Le port du Havre $\left(49^{\circ} 29^{\prime} \mathrm{N} ; 0^{\circ} 07^{\prime} \mathrm{E}\right)$ est situé à la pointe du pays de Caux, au fond de la Baie de Seine, en Manche Orientale. II est isolé de l'estuaire de la Seine. 
Le port pétrolier d'Antifer $\left(49^{\circ} 39^{\prime} \mathrm{N}\right.$; $0^{\circ} 09^{\prime} \mathrm{E}$ ) est situé à la côte, protégé par une digue longue de $3,5 \mathrm{~km}$. Le bassin principal s'ouvre largement vers le SW. Profond de $29 \mathrm{~m}$ au centre, sur la zone de manœuvre des pétroliers, il est limité par des enrochements côté digue et côté terre. Le fond sédimentaire rejoint doucement le pied des enrochements, ou une plage d'amortissement entre $-15 m$ et $-3 m$ dans les zones explorées. Au sud, un port de service est également limité par des digues avec enrochements. Le port principal et le port de service sont des bassins de marée.

Le port de Rouen (Seine-Maritime) $\left(49^{\circ} 26^{\prime} 38^{\prime \prime} \mathrm{N}\right.$; $\left.1^{\circ} 06^{\prime} 12^{\prime \prime} \mathrm{E}\right)$ se situe dans l'estuaire amont (= estuaire fluvial, haut estuaire) de la Seine. C'est un estuaire macrotidal, c'est-à-dire que la marée y est ressentie : à Rouen, le marnage est de plus de trois mètres pour un coefficient de 83 , le retard de la basse mer par rapport à l'embouchure (Honfleur, Calvados) étant de 9 heures (Dégremont \& Levêque, 2012). Les eaux y sont douces. Le bassin SaintGervais, creusé en rive droite du fleuve à sa confluence avec le Cailly, héberge le port de plaisance. Les rives, empierrées ont une pente de $45^{\circ}$. En dessous des plus basses eaux, le substrat est constitué de blocs et pierrailles irréguliers, mais toujours recouverts d'une épaisse pellicule de matières en suspension déposées, la quantité de sédiment augmentant progressivement jusqu'au fond sédimentaire constitué de vase fine. L'ensemble constitue un système lentique, à l'exception du débouché immédiat du Cailly, lotique, avec un fort courant en particulier à marée basse, qui limite le dépôt sédimentaire.

Lors de chaque plongée, tous les taxons observés identifiables avec certitude sont notés. Des prélèvements, limités aux nécessités de l'étude sont identifiés au laboratoire après la plongée et éventuellement conservés en collection de référence. Les spécimens " difficiles » ou nécessitant une compétence particulière sont soumis à des spécialistes. En plus des équipements personnels des adhérents de Port Vivant, l'association utilise les appareils de prises de vues subaquatiques suivants: Nikonos RS et Nikonos V (argentiques), Nikon D 80 en caisson (numérique), caméra vidéo Sony HDR XR 550 en caisson Mangrove. Tous les clichés utilisables pris au cours de la plongée sont analysés individuellement et tous les taxons photographiés identifiables sont ajoutés à la liste des taxons présents. À l'issue de chaque plongée, un compte rendu à diffusion interne, illustré par les clichés pris au cours de la plongée ou au laboratoire, dresse la liste des taxons observés et ajoute des commentaires (particularités biologiques, fréquence...) sur certains taxons.

Toutes les photographies illustrant cet étude ont été prises par l'auteur (@ Gérard Breton - Port Vivant), à l'exception de la Fig. 6 (@ Daniel Ingratta).

Nous n'avons pas entrepris d'étude quantitative sur la distribution des taxons dans les ports. En effet, si techniquement, nous sommes capables de positionner un cadre sur un quai, de dénombrer les individus ou taxons présents dans le quadrat et de rapporter ce décompte à une densité exprimée 
en ind. $\mathrm{m}^{-2}$, cette précision, en ce qui concerne en particulier le port du Havre, est complètement illusoire. En effet, notre expérience montre une très grande variabilité dans l'espace et dans le temps. Sur un même quai, à la même profondeur, un taxon donné, une ascidie par exemple, peut passer en quelques mètres de plusieurs centaines d'individus. $\mathrm{m}^{-2}$ à 0 ind. $\mathrm{m}^{-2}$. Cette variation longitudinale des densités se retrouve aussi verticalement sur un même quai, entre la surface et le fond, et d'un quai à l'autre (différences d'insolation) ; mais aussi au fil des saisons. C'est pourquoi nous avons privilégié une approche semi-quantitative.

\section{DÉFINITIONS ET COMMENTAIRES}

Nous appelons « espèce introduite » une espèce qui apparaît dans une région où elle n'avait pas été reconnue auparavant. "Espèce exogène, allochtone, non-indigène » [en anglais «alien species, non-indigenous species»] sont considérés ici comme synonymes. II convient d'explorer les limites sémantiques de cette définition, selon :

- l'action de l'homme. L'arrivée dans de nouveaux territoires d'une espèce donnée peut être naturelle, l'introduction stricto sensu d'une espèce est liée à l'action, volontaire ou non, de l'homme (Eno et al., 1997 ; Boudouresque, 2005). Néanmoins, beaucoup de cas restant indécis, nous appliquerons la notion d'espèce introduite sensu lato, incluant les arrivées naturelles ;

- la taille de la région considérée. Une espèce classique de la faune de la Manche Orientale peut apparaître dans les bassins du port du Havre, où elle n'avait pas été recensée auparavant. C'est le cas du polychète Bispira fabricii (Krøyer, 1856), abondant dès 2011 dans les bassins à flot ancien, et repéré seulement quelques années auparavant dans les bassins de marée par les benthologues (Dancie, com. pers.) ;

- la date d'apparition de l'espèce considérée et le succès de l'introduction. Une espèce anciennement introduite, dont l'implantation est réussie et en équilibre avec les populations antérieures, peut être considérée aujourd'hui comme faisant partie de la flore ou de la faune locales ou régionales, elle est en quelque sorte « devenue indigène ». Elle est distinguée comme « espèce naturalisée ». C'est ainsi que Haliplanella lineata (Verill), immigrée depuis le Pacifique à la fin du $19^{\mathrm{e}}$ siècle, est aujourd'hui répandue sur toutes les côtes d'Europe (Manuel, 1988), en gardant toutefois une prédilection pour le domaine paralique. Boudouresque (2005: 11) distingue, à bon escient, des introductions relativement récentes, mais antérieures à l'ère linnéenne, que seules des comparaisons d'ADN pourront mettre en évidence. II reste toutefois difficile de préciser la durée au-delà de laquelle la naturalisation, c'est-à-dire l'incorporation de l'espèce aux populations préexistantes, peut être considérée comme définitive. Nous utiliserons prudemment cette notion d'espèce naturalisée, qui nécessiterait pour 
être appliquée de fixer une limite nécessairement arbitraire à l'ancienneté d'implantation ;

- la rareté ou la méconnaissance de l'espèce considérée. La répartition géographique d'une espèce rare peut être mal connue. Une espèce donnée peut très bien être implantée antérieurement à sa première observation, voire à sa première description. Tous ces cas constituent pour nous des espèces au statut incertain [vis-à-vis de l'origine géographique réelle, de la date d'introduction, ou de la réalité de l'introduction] ; ce sont les « cryptogenic species » des auteurs anglophones (Eno et al., 1997). C'est le cas par exemple de Hydroides elegans Okuda (Zibrowius \& Thorp, 1989). Nous y ajouterons de possibles indigènes méconnues comme Hemimysis spinifera Ledoyer (Breton et al., 1996 ; Breton, 2005) ;

- le fait que l'espèce considérée se reproduit ou non dans la région où elle est nouvellement observée. Les individus d'une espèce exotique qui ne se reproduisent pas dans la région où on les observe sont qualifiés d'erratiques. Ce sont les «vagrant species» des auteurs anglophones (Eno et al., 1997), ce que l'on peut traduire littéralement par espèces vagabondes. Les observations sont en général rares, mais l'inverse n'est pas vrai : une espèce rare n'est pas obligatoirement erratique. Scyliorhinus canicula (Linnaeus) se reproduit dans le port du Havre, malgré une seule observation rapportée d'adulte (Vincent, 2001). Sont erratiques dans le port du Havre par exemple le crabe bleu Callinectes sapidus (Rathbun) (Vincent, 1987) ou encore le sar commun atlantique Diplodus sargus (Linnaeus) (Breton, sous presse), probablement arrivé dans le port du Havre par extension de son aire de répartition géographique («marginal dispersion» selon Crisp, 1958).

Au total, Boudouresque (2005: 25), passant en revue un certain nombre de critères que nous n'avons pas tous retenus parce qu'inopérants dans le cadre de notre étude naturaliste, conclut de manière pertinente qu'il est préférable de parler de probabilité d'introduction d'une espèce donnée.

Remarque. Des difficultés taxinomiques voire nomenclaturales peuvent compliquer l'histoire d'une introduction. Une Didemnum sp. invasive, repérée en janvier 1999 dans le port du Havre, est citée par Breton (2005 : 396) comme Didemnum cf. lahillei Hartmeyer. C'est une Didemnumsp. que Minchin \& Sides (2006) rapportent des ports de plaisance d'Irlande. C'est encore en nomenclature ouverte que Boudouresque (2005 : 100) l'évoque. Cette ascidie, qui a, par une coïncidence étonnante, envahi de manière simultanée plusieurs endroits de la planète (G. Lambert, com. pers.), n'aura été nommée qu'en 2002 : Didemnum vexillum Kott, avec de plus un synonyme plus récent (Didemnum vestum Kott), deux ans plus tard, par le même auteur (Lambert, 2009) (Fig. 17). Deux espèces jumelles peuvent être source de confusion. Le crabe Hemigrapsus penicillatus (de Haan) introduit en 1993 en Europe (Allemagne) a été observé 
dans le port du Havre en 1997 et publié sous ce nom (Vincent \& Breton, 1999). Cependant, une espèce jumelle Hemigrapsus takanoi Akasura et Watanabe est décrite en 2005, sur du matériel japonais (Fig. 10). La question posée, et toujours à l'étude, est de savoir si c'est cette espèce, ou bien $H$. penicillatus, (ou bien les deux !) qui a (ont) été introduite(s) en Europe (Dewarumez et al., 2011). Dans le port du Havre, tous les spécimens que nous avons examinés montrent les caractères de $H$. takanoi. Dans le domaine de l'algologie, une confusion similaire a été faite entre Caulacanthus ustulatus (Mertens) Kütz. décrite comme introduite et localement invasive en Bretagne et dans le port du Havre (Le Duff et al., 2008) et C. okamurai Yamada, espèce jumelle distinguée ultérieurement et qui est l'espèce présente en Manche.

Nous appelons « espèce invasive » une espèce qui tend à occuper de manière hégémonique le territoire, éventuellement au détriment d'occupants préexistants. Cela correspond à la définition de l'IUCN des « espèces exotiques envahissantes » (EEE) (Otero et al., 2013). II convient cependant de nuancer cette notion :

- certaines espèces sont réputées pour leurs « explosions démographiques », qui peuvent s'apparenter à des invasions localisées dans le temps et/ou dans l'espace. C'est le cas par exemple des gastropodes Onchidoris bilamellata (Linneaus) ou Odostomia scalaris (Mac Gillivray). Ces espèces sont indigènes : les notions d'espèce introduite et d'espèce invasive sont dissociées. Ces « explosions démographiques » ne sont qu'un cas extrême des fluctuations démographiques qui affectent toutes les populations naturelles ;

- la phase d'expansion qui suit l'introduction, éventuellement après une phase de latence durant laquelle la présence de l'espèce n'est pas reconnue, relève, par exemple chez Didemnum vexillum (Fig. 17), Hydroides ezoensis Okuda (Fig. 6) ou Caprella mutica Schurin (Figs. 7, 8), et pour le port du Havre, d'une expansion démographique explosive. Par la suite, l'espèce peut régresser (modèle A de Boudouresque, 2005 : 54) ou persister, avec des fluctuations (modèle $\mathrm{B}$ ). Bien sûr, la brutalité de la phase d'expansion, la périodicité des fluctuations, leur intensité, la pente du déclin éventuel, la persistance ou non d'un reliquat sont des données très variables selon les espèces et les territoires colonisés. Les deux modèles sont théoriques, tous les intermédiaires peuvent exister, et la cinétique d'une introduction d'espèce, voire le caractère invasif réel de cette espèce ne peuvent s'apprécier que sur une longue période, plus longue que la période couverte par la présente étude. La comparaison de plusieurs territoires colonisés peut être nécessaire pour apprécier le caractère invasif d'une espèce donnée, par exemple Mnemiopsis leidyi (A. Agassiz) (Antajan et al., sous presse) (Fig. 5) ;

- la nuisance d'une espèce épibenthique invasive s'appréciera aussi en fonction de son pouvoir d'épibiose (= fouling, salissure), mais aussi et surtout à ses conséquences 
sur l'écosystème où elle s'est implantée. Si elle est capable de supplanter des espèces préexistantes, son invasion se traduira par une baisse de la richesse spécifique. C'est le cas, dans le port du Havre, de Caprella mutica et Hydroides ezoensis. Au contraire, certaines espèces semblent pouvoir s'implanter sans élimination d'espèces occupant la même niche écologique. C'est le cas des deux crabes Hemigrapsus takanoi et $H$. sanguineus de Haan (Fig. 11) qui semblent s'être implantés sans variation notable des populations préexistantes de Brachyoures, bien qu'ils soient parfois soumis à des poussées démographiques importante.

\section{ESPÈCES ANCIENNEMENT INTRODUITES DANS LE PORT DU HAVRE (MANCHE ORIENTALE) (TABLEAU 1, FIGS. 1-4)}

Nous rassemblons dans l'annexe 1 l'actualisation nomenclaturale de la liste de taxons animaux du port du Havre (Breton, 2005), et dans l'annexe 2 son actualisation systématique. L'annexe 3 liste les taxons animaux repérés dans le port du Havre postérieurement à l'article de Breton (2005). Nous regroupons les données concernant les introductions anciennes (espèces naturalisées), espèces arrivées avant 1970 (limite arbitraire) sur les côtes d'Europe, dans le tableau I, suivi de remarques concernant quelques-unes de ces espèces.

\section{Ficopomatus enigmaticus (Fauvel) (Polychaeta, Sedentaria) (Figs. 2, 3)}

Le bassin Fluvial est un bassin allongé créé en 1887 , isolé des plans d'eau voisins depuis janvier 1983 par des enrochements sur lesquels une chaussée remplace les anciens ponts (Patrick Bertrand, com. pers.). Le renouvellement de l'eau, par percolation dans les enrochements et par une buse de faible diamètre, est très faible. Ce plan d'eau connaît en été des proliférations de macro- et de micro-algues, suivies d'épisodes de dystrophie, voire de dysoxie sévères. Ce fut le cas au cours de l'été 2003. Une mortalité généralisée affecta la quasi-totalité des populations de ce bassin à la seule exception de Mytilus edulis qui résista bien. Le 11 janvier 2004, 95 \% des supports libres étaient colonisés par des Ficopomatus enigmaticus, qui avaient élaboré des tubes longs de 10 à $30 \mathrm{~cm}$. C'est une espèce opportuniste, euryhaline, qui est la première à coloniser les espaces disponibles. Nous avions pu observer de telles proliférations de cette espèce dans ce même bassin au cours du printemps 1984 , soit un an après sa fermeture, avec formation de récifs à fleur d'eau mesurant jusqu'à $60 \mathrm{~cm}$ de diamètre (espèce incorrectement attribuée à Protula tubularia (Montagu) par Breton, 1981b). D'autres bioconstructions remarquables de tubes de cette espèce ont été occasionnellement observées quai de Moselle-W.

\section{Crepidula fornicata (Linnaeus) (Mollusca, Gastropoda) (Fig. 4)}

Autre opportuniste, Crepidula fornicata est présente dans les bassins à flot anciens du port du Havre, mais elle n'y prolifère jamais de manière importante. Nous avons montré que, lorsque 


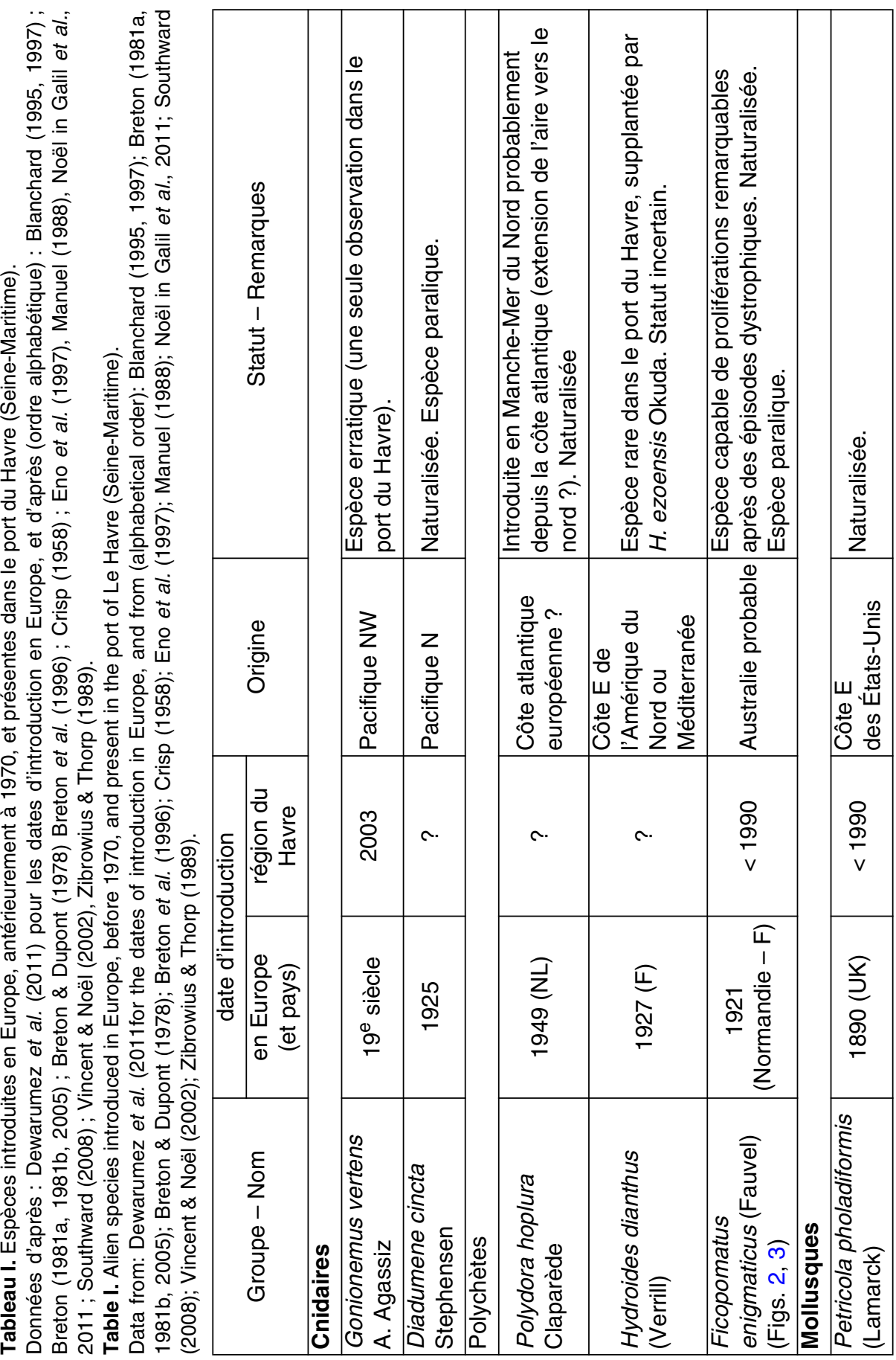




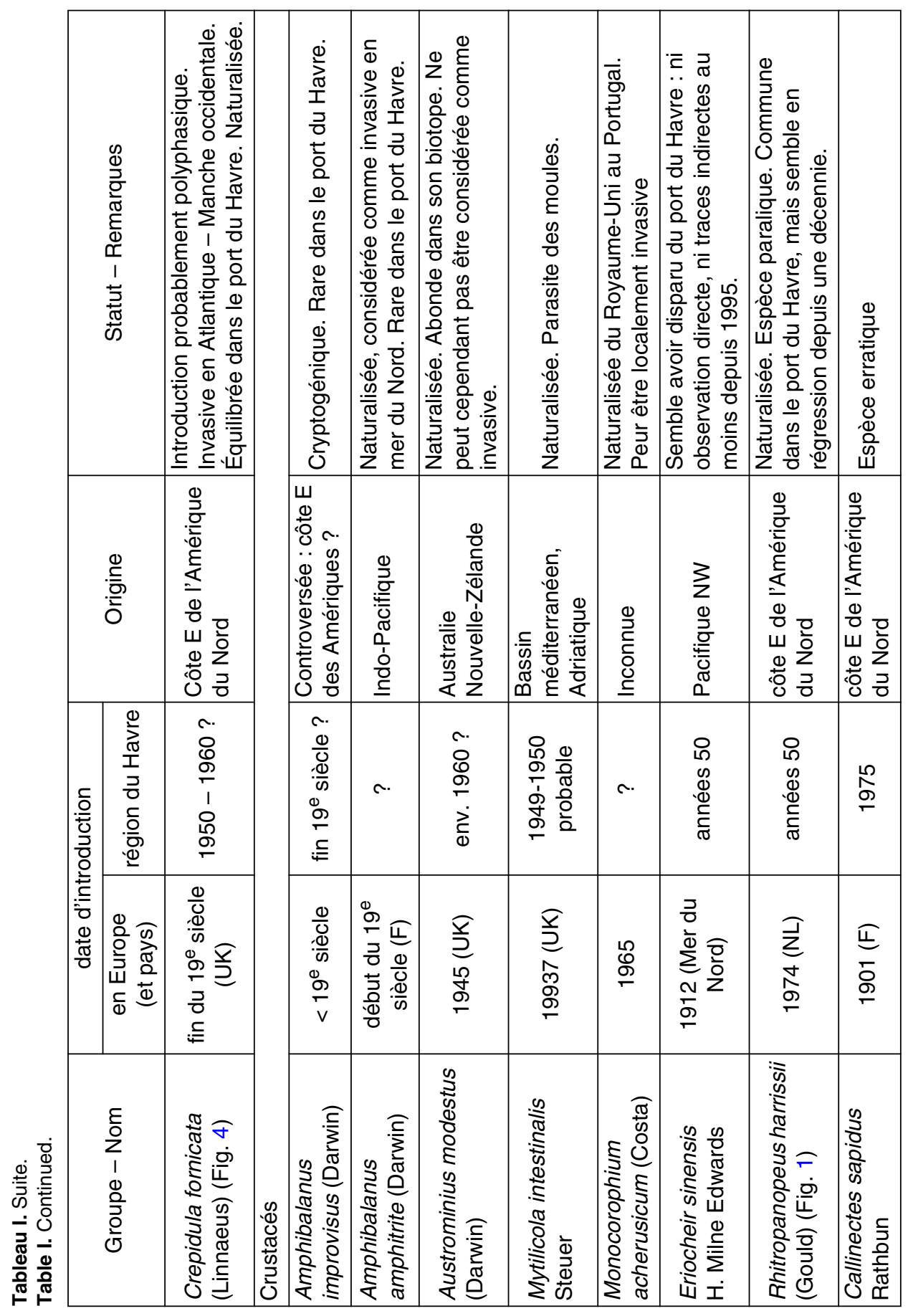




\begin{tabular}{|c|c|c|c|c|c|c|c|}
\hline & 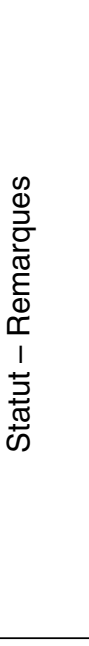 & & 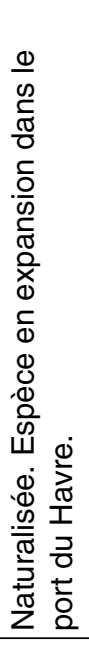 & 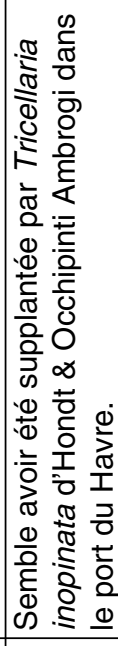 & & 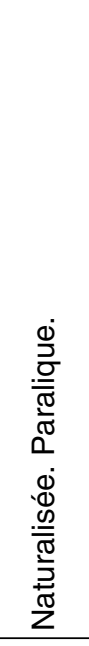 & 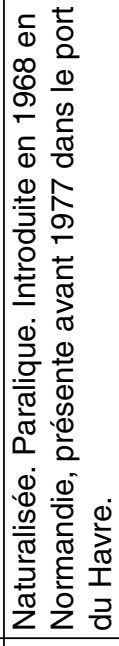 \\
\hline & 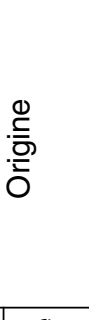 & & 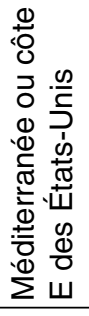 & 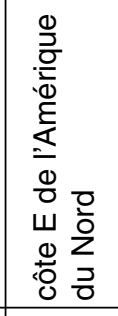 & & 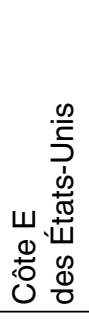 & 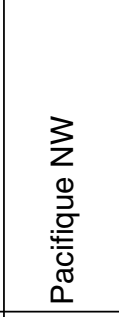 \\
\hline 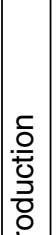 & 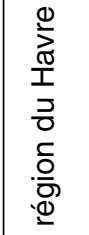 & & $\sim$ & $\frac{\infty}{\stackrel{\infty}{\sigma}}$ & & $\begin{array}{l}\infty \\
\stackrel{\infty}{\circ} \\
\stackrel{v}{v}\end{array}$ & $\stackrel{\infty}{\circ}$ \\
\hline 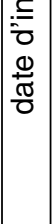 & 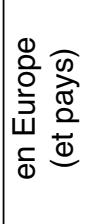 & & 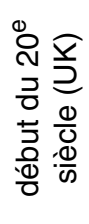 & \begin{tabular}{l}
$\widehat{Y}$ \\
$己$ \\
$\varnothing$ \\
$\varnothing$ \\
\hdashline
\end{tabular} & & 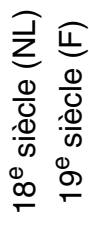 & 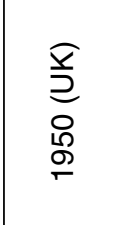 \\
\hline & 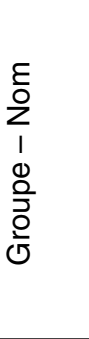 & 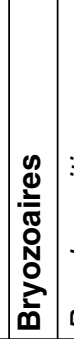 & 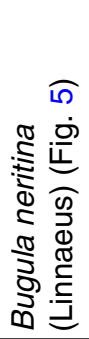 & 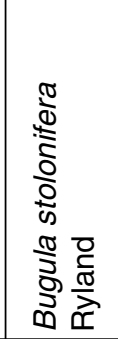 & 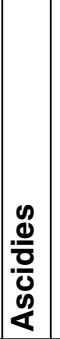 & 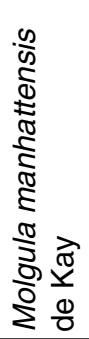 & 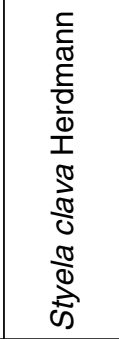 \\
\hline
\end{tabular}




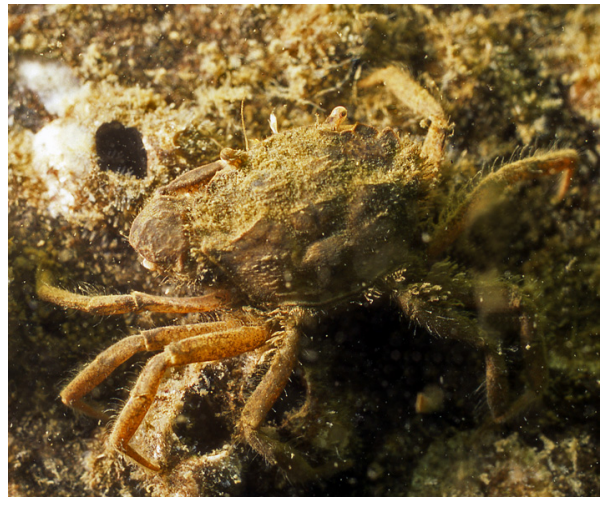

Fig. 1. Rhitropanopeus harrissii (Gould). Quai de Moselle, port du Havre, 04.02.2008.

Fig. 1. Rhitropanopeus harrissii (Gould). Moselle quay, port of Le Havre, 04.02.2008.

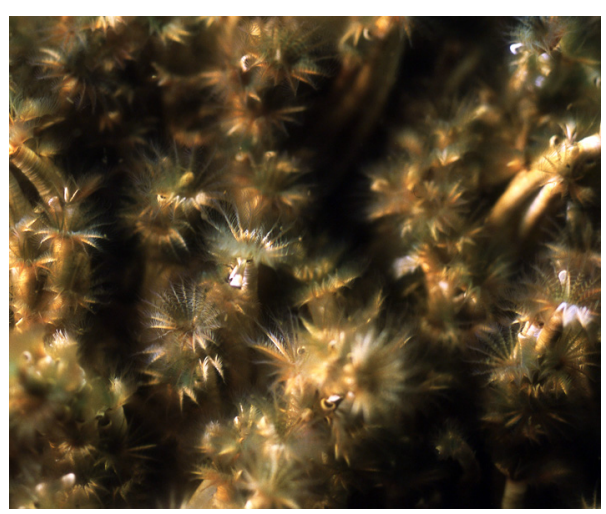

Fig. 2. Ficopomatus enigmaticus (Fauvel). Bassin Fluvial, port du Havre, population dense après un épisode de dystrophie, 24.08.2008.

Fig. 2. Ficopomatus enigmaticus (Fauvel). Fluvial basin, port of Le Havre, dense population after a dystrophic event, 24.08.2008.

les conditions trophiques sont défavorables, $C$. fornicata peut, à l'âge adulte, abandonner sa stratégie trophique de suspensivore, et devenir temporairement brouteuse (Breton \& Huriez, 2010).

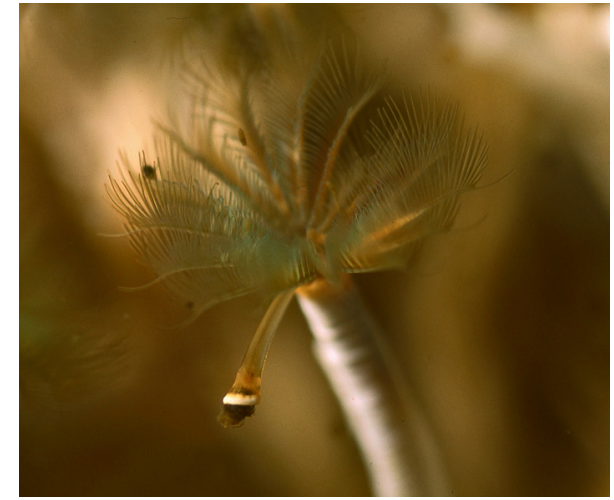

Fig. 3. Ficopomatus enigmaticus (Fauvel). Bassin Fluvial, port du Havre, comme figure 2, détail, 24.08.2008.

Fig. 3. Ficopomatus enigmaticus (Fauvel). Fluvial basin, port of Le Havre, as Figure 2, detail, 24.08.2008.

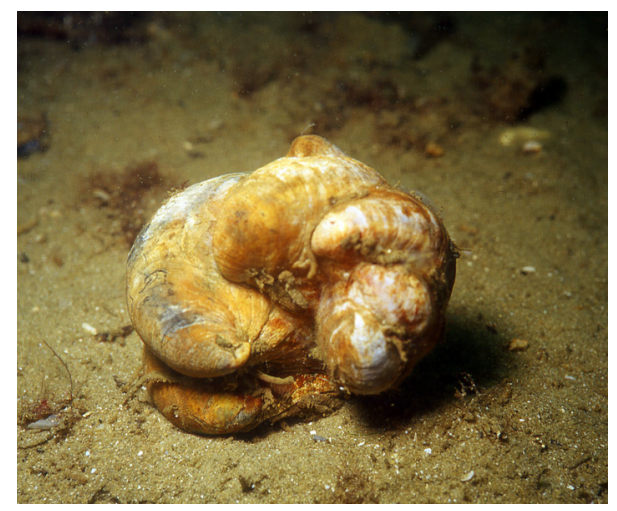

Fig. 4. Crepidula fornicata (Linnaeus). Bassin Vauban, port du Havre, 08.10.2006.

Fig. 4. Crepidula fornicata (Linnaeus). Vauban basin, port of Le Havre, 08.10.2006.

\section{Eriocheir sinensis H. Milne Edwards (Crustacea, Decapoda)}

Le crabe chinois était assez fréquent jadis dans les bassins et canaux de l'arrière-port (Vincent, 1996), à tel point 
que, dans les années 50 et 60 , il était encore considéré comme nuisible. Entre 1952 et 1990, 33 captures ont été recensées dans le port du Havre (Vincent, 1996 ; Vincent \& Noël, 2002). En 1995 et 1996, des plongées ont été organisées pour rechercher des indices de sa présence, en vain. Depuis, aucune observation n'a été rapportée. Il est possible qu'il ait disparu.

\section{INTRODUCTIONS RÉCENTES DANS LE PORT DU HAVRE (MANCHE ORIENTALE) (FIGS. 5-17)}

Les espèces animales épibenthiques ou planctoniques introduites dans le port du Havre après 1970, et reconnues en plongée subaquatique, sont présentées dans l'ordre systématique.

\section{Haliclona xena de Weerdt (Porifera, Demospongia)}

Statut incertain: indigène méconnue?

Premières observations dans le port du Havre en 1993, 1994 et 1995, dans le bassin Fluvial, où elle abonde. Elle semble avoir disparu de ce bassin hormis une observation en 2000. Elle a été rencontrée, rarement, dans les bassins à flot anciens entre 1997 et 2009. L'espèce a été décrite en 1986 (de Weerdt, 1986), de plans d'eau paraliques des Pays-Bas. Elle peut avoir existé en Manche - Mer du Nord antérieurement à sa description, et avoir été confondue avec une autre espèce, par exemple Haliclona cinerea (Grant) qui est morphologiquement très variable en domaine paralique (obs. pers.). Son statut, espèce introduite vs. espèce indigène méconnue reste donc incertain.

\section{Mnemiopsis leidyi (A. Agassiz) (Ctenophora, Nuda) (Fig. 5)}

Espèce introduite, modérément invasive dans le port du Havre.

Première observation le 29 mai 2005, bassin Vauban - Est identification confirmée sur photo : Fig. 5). Confondue à l'époque avec l'espèce indigène Bolinopsis infundibulum Müller, ce n'est qu'en 2010 que l'identification a été rectifiée. Néanmoins, de nombreux clichés photographiques permettent d'authentifier la présence de $M$. leidyi chaque année à la belle saison, les premières observations de l'année se faisant entre mai et octobre, entre 2005 et 2011. L'espèce n'a pas été observée en 2012. Elle se rencontre dans tous les plans d'eau du port du Havre, entre les zones II et IV du paralique. Les populations peuvent être très denses dans les bassins à flot anciens, une plongée a permis d'évaluer la densité à une à plusieurs centaines d'individus par $\mathrm{m}^{3}$. Sa présence dès 2005 dans le port du Havre a conduit Antajan et al. (sous presse) à nuancer les voies d'arrivée de ce cténaire sur les côtes françaises.

\section{Hydroides ezoensis Okuda (Polycheta, Sedentaria) (Fig. 6)}

Espèce introduite et invasive.

Lors de la première observation, le 23 février 1997, sur le quai d'un bassin à flot ancien, les individus sont distants en moyenne de 0,5 à $1 \mathrm{~m}$. Une démographie «explosive » dans le courant de l'été 1997 a fait que, à l'automne 


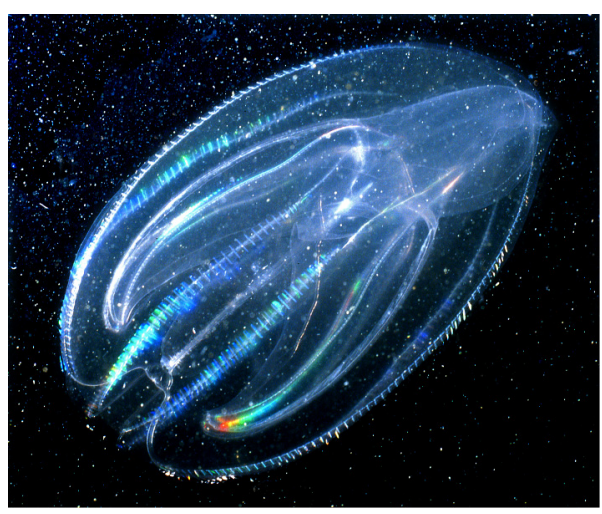

Fig. 5. Mnemiopsis leidyi (A. Agassiz). Bassin Vauban, port du Havre, première observation, 25.09.2005, photo en aquarium.

Fig. 5. Mnemiopsis leidyi (A. Agassiz). Vauban basin, port of Le Havre, first observation, 25.09.2005, photo in an aquarium.

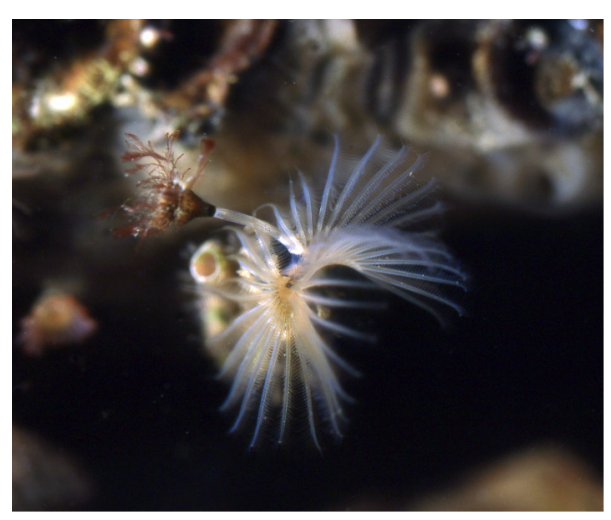

Fig. 6. Hydroides ezoensis Okuda. Bassin Vauban, port du Havre, 19.04.2009. Les balanes en activité à l'arrière-plan sont Balanus crenatus Bruguière. Cliché Daniel Ingratta.

Fig. 6. Hydroides ezoensis Okuda. Vauban basin, port of Le Havre, 19.04.2009. The active barnacles in the background are Balanus crenatus Bruguière. (C) Daniel Ingratta.

1997, il était impossible de prendre un cliché (surface photographiée $24 \times$ $36 \mathrm{~mm}$ ) d'un Hydroides ezoensis isolé : chaque photo montrait deux à six tubes (Vincent \& Breton, 1999). Depuis, l'espèce se retrouve, avec des densités comparables, dans tous les bassins à flot anciens, et dans plusieurs bassins de marée (zones II et III (IV) du paralique). L'espèce, d'origine japonaise, avait été introduite accidentellement dans la décennie 70 avec du naissain d'huitres japonaises Crassostrea gigas (Thunberg), mais l'espèce ne s'est ni implantée, ni disséminée à partir de ce foyer atlantique (Gruet et al., 1976 ; Zibrowius, 1978 ; Gruet \& Baudet in Dauvin, 1997). De 1977 à 1982, l'espèce a colonisé l'estuaire de la Solent dans le sud de l'Angleterre, l'estuaire de la Hamble, Southampton et Portsmouth (Thorp et al., 1987). La population havraise de Hydroides ezoensis trouve son origine dans celle du sud de l'Angleterre; les fondateurs ayant possiblement voyagé sur la coque des ferries transmanche. Depuis son implantation, nous n'avons observé dans les bassins du port du Havre aucun autre Hydroides spp.: $H$. dianthus (Verrill), $H$. elegans (Haswell) et $H$. norvegica (Gunnerus) restaient certes rares, mais toutes leurs observations datent d'avant l'arrivée de $H$. ezoensis, qui s'avère donc une espèce invasive dans le port du Havre.

\section{Ensis directus (Conrad) (Mollusca, Bivalvia) (Mollusca, Bivalvia)}

Espèce introduite, non invasive (?) dans le port du Havre.

Une coquille vide mais fraîche recueillie bassin du Commerce le 14 décembre 2003 a permis de reconnaître la présence de l'espèce dans le port du Havre. Depuis, l'espèce a été 
retrouvée dans différents plans d'eau : bassins à flot anciens, bassins de marée (darse du Pacifique) et bassins à niveau constant de l'arrière-port. Dans la darse de l'Océan, des populations denses, détectées en plongée par leurs siphons, sont repérées sous quelques décimètres d'eau. C'est à cause de cette prolifération, pour l'instant localisée, que nous avons tempéré d'un point d'interrogation son statut de non invasive dans le port du Havre.

\section{Ruditapes philippinarum (Adams \& Reeves) (Mollusca, Bivalvia)}

Espèce introduite, non invasive dans le port du Havre. Une seule observation, le $1^{\text {er }}$ juin 2010 , d'une palourde japonaise vivante dans un bassin à flot ancien, un an environ après son observation dans le port de Dunkerque (Yves Müller, com. pers. ; Dewarumez et al., 2011). L'absence d'observation ultérieure indique, soit la rareté de l'espèce dans le port du Havre, soit l'échec de son implantation.

\section{Crassostrea gigas (Thunberg) (Mollusca, Bivalvia)}

Espèce introduite (considérée comme naturalisée par Lapègue et al., 2006), non invasive dans le port du Havre.

Repérée depuis 2008, principalement dans les bassins de marée (port de plaisance, Forme VII, darse du Pacifique), mais aussi dans un bassin à flot ancien (un individu : bassin de la Citadelle), Crassostrea gigas existait certainement avant les premières observations. Elle coexiste avec l'espèce indigène Ostrea edulis
Linnaeus qui semble confinée aux bassins de marée. Comme le soulignent Poppe \& Goto (1993 : 79) « The correct identification of Ostreidae is difficult: especially in this century, the classic native European species has been mixed up with imported oysters from all over the world " [L'identification correcte des Ostreidae est difficile : en particulier au cours de ce siècle, l'espèce indigène européenne classique s'est trouvée mélangée avec des huitres importées de l'ensemble de la planète]. La distinction des deux espèces dans le port du Havre est donc particulièrement ingrate, et la coexistence de caractères de l'une et l'autre espèce dans un même individu laisse entrevoir des possibilités d'hybridation plus importantes que ne le laisserait penser une distance systématique générique. II est à noter que Ostrea edulis avait fait l'objet d'une introduction volontaire dans un bassin à flot ancien du centre-ville (Breton, 2005).

\section{Hemimysis spinifera Ledoyer (Crustacea, Mysidacea)}

Statut incertain : indigène méconnue? Non invasive.

Observée dans un aqueduc non fonctionnel du bassin Fluvial dès le printemps 1992, photographiée et collectée en mai 1993 (Breton et al., 1996), l'espèce sera identifiée par J.-P. Lagardère. En 1996, l'espèce n'était connue que de la grotte du Grand Salon, côte de l'Algarve, Portugal, d'où Ledoyer (1989) l'avait décrite. L'espèce a-t-elle une aire de répartition disjointe Algarve - Normandie, ou a-t-elle migré d'une région à l'autre ? 
Cela apparaît hautement improbable, compte tenu du mode de vie de ce crustacé. A-t-elle une aire de répartition plus large, incluant l'Algarve et la Normandie, mais en restant confinée aux environnements sous-marins obscurs (grottes sous-marines obscures ou bien leurs équivalents artificiels comme l'aqueduc du bassin Fluvial) ? $\mathrm{Y}$ a-t-il eu migration ou introduction ? Si oui, par quel vecteur? Le doute qui entoure ces questions nous a conduit à attribuer à Hemimysis spinifera un statut incertain.

\section{Caprella mutica Schurin (Crustacea, Amphipoda) Figs. 7, 8)}

Espèce introduite et invasive.

Les premières observations sûres, avec capture et identification datent d'octobre 2004, dans des bassins à flot anciens. Depuis, l'espèce est régulièrement observée dans les zones II et III du paralique, c'est-à-dire les bassins de marée et les bassins à flot anciens. Dans ces derniers, elle est sujette à des épisodes de prolifération particulièrement impressionnants entrecoupés d'épisodes où l'espèce est très discrète. Cependant, l'espèce pourrait avoir été présente plus tôt dans les bassins à flot anciens du port du Havre, puisqu'une photo de Halichondria bowerbanki Burton du 16 octobre 1994 (quai Hermann du Pasquier - E) montre plusieurs dizaines de jeunes caprelles sur un rameau de l'éponge, avec une taille et un habitus qui évoquent très fortement les population de juvéniles de Caprella mutica (Breton et al., 1996 : Fig. 8, p. 14). Malheureusement, aucun échantillon n'a été conservé. C. mutica a donc réussi dans le port du Havre une implantation pionnière pour la Manche et constitue, à ce jour, l'introduction la plus méridionale de l'espèce (Cook et al., 2007 ; Dewarumez et al., 2011).

Depuis 2004, Caprella mutica semble avoir supplanté les espèces indigènes de caprelles: Caprella equilibra Say et Phtisica marina Slabber qui avaient été observées précédemment dans les bassins à flot anciens, et qui n'ont pas été revues. Seule, une Caprella linearis (Linnaeus), espèce qui n'avait pas été recensée précédemment, a été recueillie dans le bassin Fluvial où Caprella mutica ne prolifère pas.

\section{Palaemon macrodactylus Rathbun (Crustacea, Decapoda) (Fig. 9)}

Espèce introduite, non invasive.

Signalé dès l'été 2005 dans le port du Havre (Thierry Vincent, com. pers.). Quelques individus ont pu être observés, photographiés ou capturés à partir de juin 2007 en zone IV du paralique. Cette colonisation est pionnière pour la Manche (Lavesque et al., 2010). Les individus restent rares, difficiles à observer. L'espèce n'ayant pas été revue depuis 2010, la question du succès ou de l'échec de son implantation est posée.

\section{Hemigrapsus takanoi Asakura \& Watanabe (Crustacea, Decapoda)} (Fig. 10)

Espèce introduite, non invasive.

La première observation de cette espèce de crabe est faite le 26 octobre 1997, dans une écluse en zone III du 


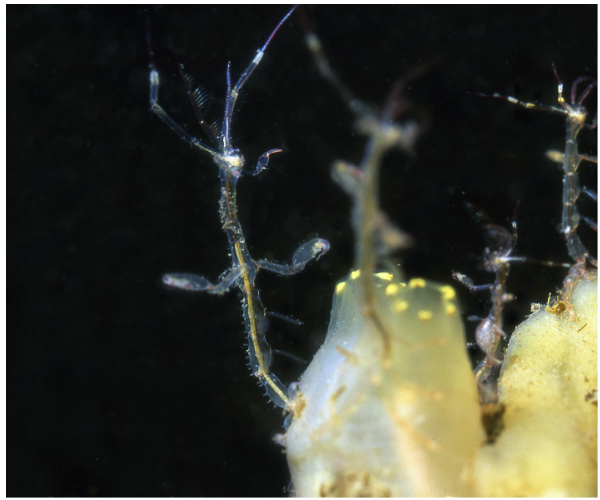

Fig. 7. Caprella mutica Schurin. Deux mâles et une femelle, agrippés à une ascidie Ciona intestinalis, bassin de la Barre, port du Havre, 05.10.2012.

Fig. 7. Caprella mutica Schurin. Two males and one female clinged on to an ascidian Ciona intestinalis. Barre basin, port of Le Havre, 05.10.2012.

paralique (Vincent \& Breton, 1999). Dès 1998 , on le rencontre dans tous les plans d'eau du port du Havre, alors qu'il ne se trouve pas sur les côtes normandes de la Manche de part et d'autre du Havre : son introduction dans le port du Havre apparaît donc comme pionnière. Elle pourrait trouver son origine dans des populations déjà établies dans la région de La Rochelle (Charente-Maritime) depuis 1994 (Noël et al., 1997). En effet, le port du Havre, en 1995 et 1997, a accueilli les navires de la "Transat Jacques Vabre » Le Havre - Carthagène, dont certains arrivaient de La Rochelle, qui pouvaient transporter les larves de $H$. takanoi dans leurs eaux de ballast.

II convient de remarquer que c'est sous l'identité de $H$. penicillatus de Haan que les premières mentions de ce crabe sur les côtes européennes ont été rapportées (Noël et al., 1997 ; Gollash, 1999 ; Vincent \& Breton,

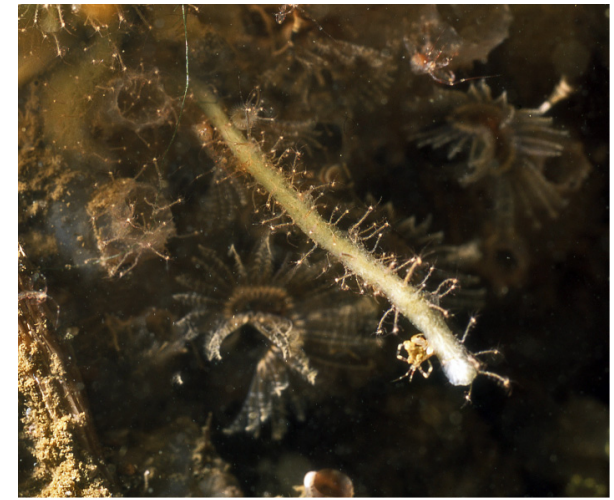

Fig. 8. Caprella mutica Schurin. Nombreux juvéniles accrochés au rameau (diamètre env. $3 \mathrm{~mm}$ ) d'un spongiaire Halichondria bowerbanki. Les panaches d'Hydroides ezoensis, à l'arrièreplan, donnent une idée de l'échelle. Bassin de la Citadelle, port du Havre, 14.08.2010.

Fig. 8. Caprella mutica Schurin. Numerous juveniles clinged on to the branch (diameter ca. $3 \mathrm{~mm}$ ) of a sponge Halichondria bowerbanki. The branchial crowns of Hydroides ezoensis in the background, give an idea of the scale. Citadelle basin, port of Le Havre, 14.08.2010.

1999 ; Nijland \& Beckman, 2000 ; d'Udekem d'Acoz \& Faasse, 2002), l'espèce $H$. takanoi n'ayant été décrite et distinguée de son espèce jumelle H. penicillatus qu'en 2005 (Asakura \& Watanabe, 2005). Comme tous les spécimens des côtes d'Europe examinés depuis, les Hemigrapsus du port du Havre appartiennent tous à l'espèce H. takanoi (Noël, 2011). Mais la vigilance doit demeurer, une introduction de l'espèce $H$. penicillatus n'étant pas exclue.

Hemigrapsus takanoi semble s'être implantée dans le port du Havre en équilibre avec les espèces qu'elle aurait pu concurrencer : Carcinus maenas (Linnaeus), Necora puber (Linnaeus) et Hemigrapsus sanguineus de Haan, introduit après $H$. takanoi. 


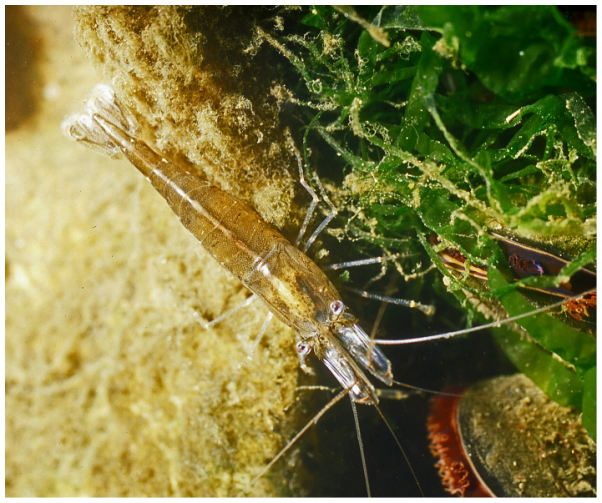

Fig. 9. Palaemon macrodactylus Rathbun. Quai de Moselle, port du Havre, 13.07.2008.

Fig. 9. Palaemon macrodactylus Rathbun. Moselle quay, port of Le Havre, 13.07.2008.

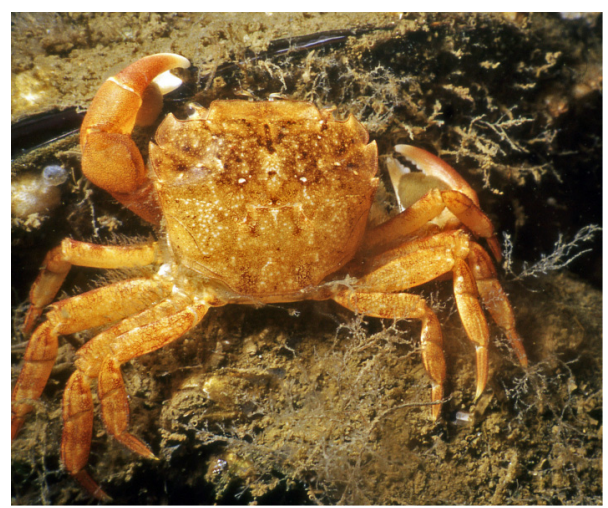

Fig. 10. Hemigrapsus takanoi Asakura \& Watanabe. Mâle. Bassin Vauban, port du Havre, 08.10.2005.

Fig. 10. Hemigrapsus takanoi Asakura \& Watanabe. Male. Vauban basin, port of Le Havre, 08.10.2005.

\section{Hemigrapsus sanguineus de Haan (Crustacea, Decapoda) (Fig. 11)}

Espèce introduite, non invasive.

Après une première observation le 29 août 1999, à basse mer dans un

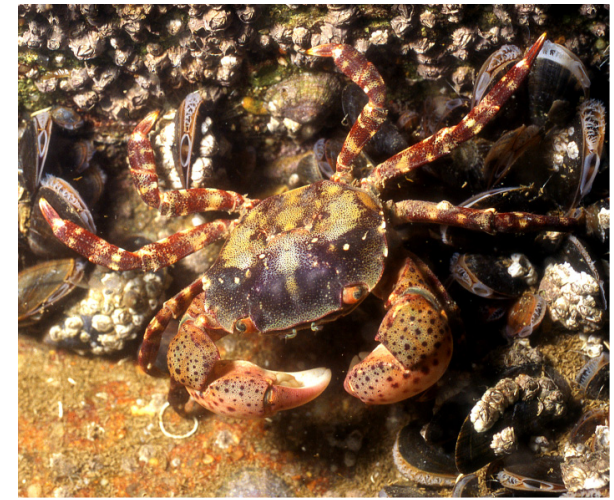

Fig. 11. Hemigrapsus sanguineus de Haan. Mâle. Digue Sud, port du Havre, 28.07.2011.

Fig. 11. Hemigrapsus sanguineus de Haan. Male. South dyke, port of Le Havre, 28.07.2011.

bassin de marée, d'une population abondante d'Hemigrapsus sanguineus, une seconde est repérée en plongée dans un bassin à niveau constant de l'arrière port (Breton et al., 2002). Depuis, l'espèce est rencontrée dans tous les types de plans d'eau du port, de la zone II à la zone IV du paralique. II peut constituer des populations abondantes ou au contraire rester extrêmement discret sans que nous puissions actuellement expliquer ces variations apparentes de densité de population («apparentes " parce que nous pensons qu'il s'agit de variations de visibilité de ces crabes en plongée plus que de variations démographiques). Dans les sites où $H$. sanguineus abonde, son implantation semble s'être faite de manière équilibrée avec les espèces de Crustacés décapodes avec lesquelles elle coexiste: Carcinus maenas, Hemigrapsus takanoi, Necora puber, et le plus rare Pilumnus hirtellus (Linnaeus). En ce qui concerne le port du Havre, où l'espèce semble avoir réussi une implantation pionnière (pour 
la Manche), nous ne partageons donc pas l'avis de Dewarumez et al. (2011) sur le caractère invasif et les lourds impacts sur l'habitat et les espèces natives, ainsi que sur l'homme et ses activités liés à l'introduction de $\mathrm{Hemi}$ grapsus sanguineus.

\section{Tricellaria inopinata} d'Hondt \& Occhipinti Ambrogi (Bryozoa, Cheilostomata) (Fig. 12)

Espèce introduite, invasive dans le port du Havre.

Découverte dans la lagune de Venise (Adriatique) en 1982, décrite et nommée en 1985 (d'Hondt \& Occhipinti Ambrogi, 1985), l'espèce a, entre 1996 et 2001, envahi la façade atlantique de l'Europe et la mer du Nord. Cette espèce n'était pas encore reconnue sur les côtes françaises de la Manche et de la mer du Nord lorsque, le 12 janvier 2003, elle a été repérée dans le sas Fluvial (bassin à flot ancien, zone III du paralique) ; depuis, sa présence est avérée dans presque tous les bassins du port du Havre, de la zone II à la zone IV du paralique. Elle y est très abondante et y a supplanté les populations de Bugula stolonifera Ryland dont Breton (2005) disait « Espèce paralique, annuelle, le bryozoaire le plus commun du port du Havre». Peut connaître des " explosions » démographiques ". L'implantation et le développement de $T$. inopinata, en parallèle avec le développement de Bugula neritina (Linnaeus) a vu la décroissance, voire la disparition, localement, de B. stolonifera. Tricellaria inopinata est donc une espèce invasive, comme elle l'a été dans la lagune de Venise, pendant une période «de prospérité qui a duré une quinzaine

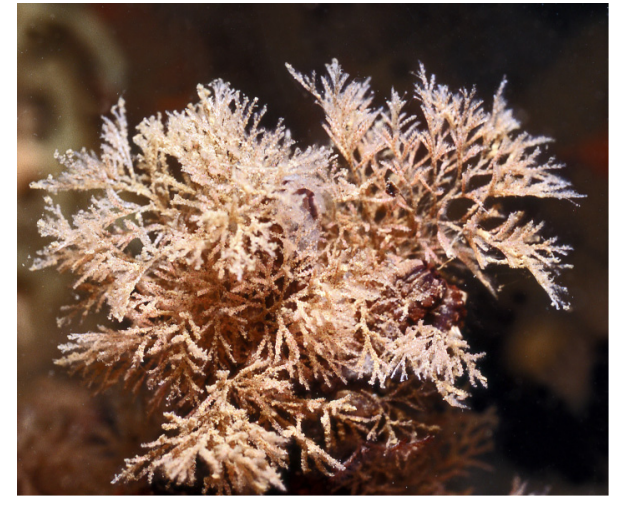

Fig. 12. Tricellaria inopinata d'Hondt \& Occhipinti Ambrogi. Bassin de la Barre, port du Havre, 14.10.2011. Photo également publiée dans Otero et al. (2013, p. 24).

Fig. 12. Tricellaria inopinata d'Hondt \& Occhipinti Ambrogi. Barre basin, port of Le Havre, 14.10.2011. Picture also published by Otero et al. (2013, p. 24).

d'années ", avant d'entrer en régression (Breton \& d'Hondt, 2005).

Remarque. Bugula neritina (Fig. 13) est considérée comme une espèce introduite, mais dont l'origine reste discutée; $B$. stolonifera est également considérée comme introduite, provenant de la côte est de l'Amérique du Nord. Tricellaria inopinata provient de la côte ouest de l'Amérique du Nord où elle avait été confondue avec une autre espèce Tricellaria occidentalis (Trask, 1873), et de la mer du Japon (d'Hondt et al., 2004).

\section{Fenestrulina delicia Winston, Hayward \& Craig (Bryozoa, Cheilostomata)}

Espèce introduite, probablement non invasive dans le port du Havre. 


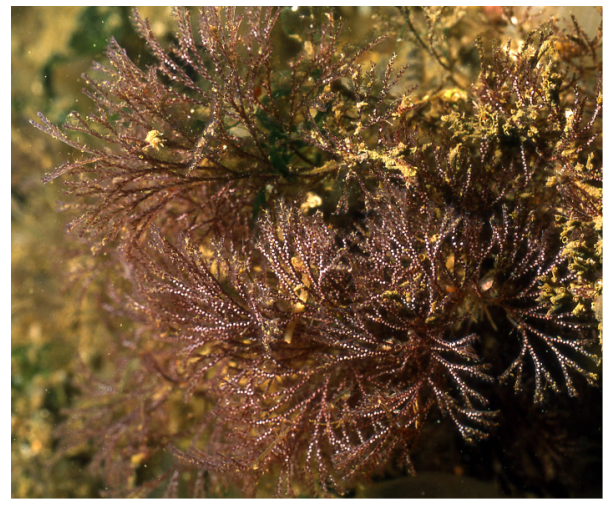

Fig. 13. Bugula neritina (Linnaeus). Bassin de la Citadelle, port du Havre, 14.08.2010.

Fig. 13. Bugula neritina (Linnaeus). Citadelle basin, port of Le Havre, 14.08.2010.

Une seule - petite - colonie recueillie le 16 juin 2013 dans un bassin de marée, le bassin du Commerce, au pied du quai George V sur un verre, avec Hydroides ezoensis, Haliclona cinerea Grant, Cryptosula pallasiana (Moll), Conopeum reticulum (Linnaeus), Lagotia viridis Wright et Balanus crenatus Bruguière. Elle a été distinguée de l'espèce voisine par la présence de 1 à 4 épines orales (De Blauwe, 2009). L'espèce est originaire de la côte orientale (où elle a été décrite) ou occidentale des États-Unis. Elle est observée en 2005 aux Pays-Bas, puis en 2007 et 2008 en Manche occidentale (De Blauwe, 2009 ; Dewarumez et al., 2011). Elle avait été repérée le 25 septembre 2010 dans le port d'Antifer. Ces deux observations viennent donc combler un hiatus entre les signalisations en Manche occidentale et en Mer du Nord.

Ces deux observations - récentes d'une espèce discrète et qui peut facilement passer inaperçue n'autorisent pas plus de précision sur son statut.
Perophora japonica Oka (Tunicata, Ascidiacea)

Espèce introduite, non invasive.

Repérée pour la première fois le 24 août 1996 dans un bassin de marée, cette petite ascidie sociale, dont le territoire d'origine est le Pacifique nordouest, est assez rarement observée dans les bassins à flot anciens et dans les bassins de marée. Elle peut échapper à la vigilance des plongeurs et être plus fréquente que ne le laisseraient supposer ses observations. Monniot \& Monniot (1985) signalent son apparition dès 1982 à Saint-Vaast-la-Hougue (Manche) (Goulletquer etal., 2002). Elle est connue en Bretagne à Concarneau (Finistère) (Bay-Nouailhat, 2007) et, sur la côte sud de l'Angleterre à Plymouth depuis 1999 (Nishikawa et al., 2000) et dans le Fleet (Dorset) (Bishop, 2005). Cette répartition témoigne d'une préférence pour le domaine paralique.

Elle n'est assurément pas invasive.

\section{CorellaeumyotaTraustedt(Tunicata, Ascidiacea) (Fig. 14)}

Espèce introduite, non invasive.

Après une première observation le 22 juillet 2007 dans un bassin à flot ancien (Françoise Monniot, com. pers., août 2007), cette ascidie, retrouvée la même année en abondance dans le port de Fécamp (4 novembre 2007), est régulièrement rencontrée depuis dans certains bassins de marée, sous les pierres au niveau des très basses mers, ou sur les quais des bassins à flot anciens. Elle n'est jamais très abondante. Originaire du domaine antarctique et subantarctique (Monniot, soumis), elle est reconnue dans les zones tempérées de l'hémisphère nord dès 


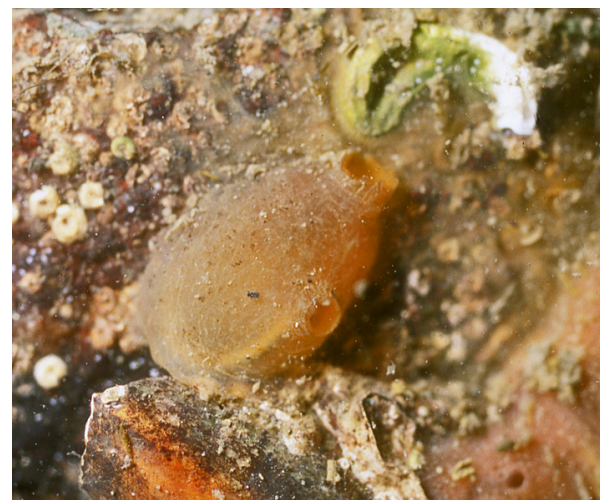

Fig. 14. Corella eumyota Traustedt. Forme VII, port du Havre, 05.06.2010. Sous une pierre qui hébergeait Hydroides sp., des spirobes et Haliclona cinerea.

Fig. 14. Corella eumyota Traustedt. Dry dock VII, port of Le Havre, 05.06.2010. Under a stone which harboured Hydroides sp., spirobes and Haliclona cinerea.

2002 en Bretagne (Lambert, 2004), puis en 2004 sur la côte sud de l'Angleterre (Arenas et al., 2006). II est possible que cette espèce, qui affectionne les ports, soit plus largement répartie, mais sous-estimée. Dans le port du Havre, elle n'est pas invasive.

\section{Botrylloides violaceus Oka (Tunicata, Ascidiacea) (Figs. 15, 16)}

Espèce introduite, non invasive.

Le 26 août 2005, une plongée était organisée pour recueillir et remettre à Gretchen Lambert des Didemnum vexillum Kott, dont elle étudiait l'expansion en différents points du globe. Dans les échantillons remontés, elle a reconnu une autre synascidie introduite, Botrylloides violaceus Oka, dans deux bassins à flot anciens. Cette identification a été confirmée par Françoise Monniot (com. pers., 02.11.2005).
L'espèce, qui affiche des couleurs vives, n'aurait pas pu échapper à la vigilance des plongeurs, ce qui indique une introduction récente dans le port du Havre, en 2004 ou 2005. Originaire du Pacifique occidental, cette espèce a colonisé les côtes ouest, puis est de l'Amérique du Nord entre 1977 et 1992, l'Adriatique (Venise) en 1993, la mer du Nord et la Manche occidentale en 2000, la Grande Bretagne et le Belgique en 2004 (Dewarumez et al., 2011). Sa date d'introduction dans le port du Havre est donc cohérente avec le schéma de son expansion.

Depuis 2005, elle est présente dans les bassins de marée et les bassins à flot anciens (zones II et III du paralique), et dans la zone IV en moins grande quantité. II arrive qu'elle connaisse des développements importants sans jamais être réellement invasive.

\section{Didemnum vexillum Kott (Tunicata, Ascidiacea) (Fig. 17)}

Espèce introduite et invasive.

Cette ascidie a été observée pour la première fois le 13 décembre 1998, dans la partie SW du bassin Vauban, bassin à flot ancien, où elle recouvrait 10 à $20 \%$ de la surface du quai où cette population de petite taille s'était implantée. Les autres quais semblaient indemnes. Le 19 octobre 1999, les quais de la partie ouest du bassin Vauban, sur plusieurs centaines de mètres de long, sur toute leur hauteur, étaient recouverts avec un taux de recouvrement de plus de $100 \%$ (autorecouvrement). Le développement de cette ascidie ne respectait que les siphons des autres ascidies, par 


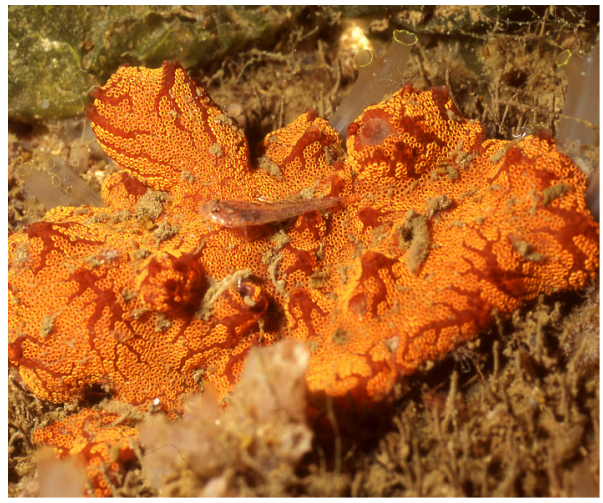

Fig. 15. Botrylloides violaceus Oka. Bassin de la Barre, port du Havre, 10.07.2011. Le poisson est Pomatoschistus pictus (Malm).

Fig. 15. Botrylloides violaceus Oka. Barre basin, port of Le Havre, 10.07.2011. The fish is Pomatoschistus pictus (Malm).

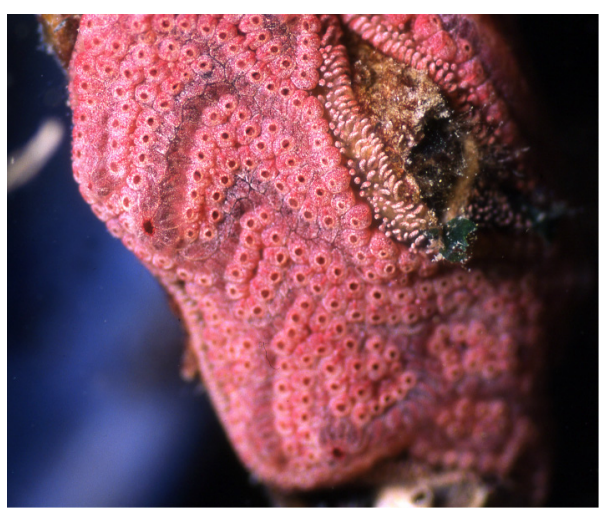

Fig. 16. Botrylloides violaceus Oka. Bassin de la Barre, port du Havre, 07.12.2008. Détail.

Fig. 16. Botrylloides violaceus Oka. Barre basin, port of Le Havre, 07.12.2008. Detail.

exemple Styela clava, l'ouverture des moules, et parfois l'oscule d'une éponge. Didemnum vexillum était observée, certes avec des densités moins impressionnantes, dans d'autres bassins à flot. Dès 2002-2003, l'espèce est à la fois en expansion

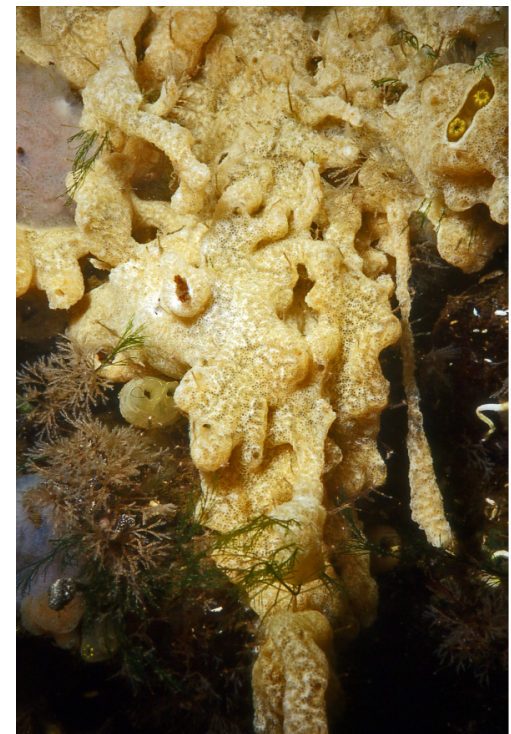

Fig. 17. Didemnum vexillum Kott. Bassin Vauban, port du Havre. 08.10.2006. Noter les Ciona intestinalis presque complètement recouvertes, et Tricellaria inopinata.

Fig. 17. Didemnum vexillum Kott. Vauban basin, port of Le Havre. 08.10.2006. Note the nearly completely overgrown Ciona intestinalis and Tricellaria inopinata.

(bassins de marée, plans d'eau à niveau constant de l'arrière-port) et en régression : elle affiche des densités et des taux de recouvrement beaucoup plus faibles quoique fort variables d'une saison à l'autre. G. Lambert (com. pers., 2005) signale que, par une coïncidence étonnante et encore inexpliquée, l'espèce a connu une augmentation spectaculaire de biomasse et de nouvelles introductions de manière quasi simultanée en différents endroits de la planète (Nouvelle-Zélande, États-Unis, ...).

II faut remarquer que cette ascidie a été repérée, et son caractère invasif souligné, avant d'être définitivement identifiée à l'espèce décrite par Kott 
(2002) (Lambert, 2009 ; Stefaniak et al., 2009). Auparavant, elle avait été rapprochée de $D$. lahillei Hartmeyer; $D$. helgolandicum Michaelsen; $D$. lutarium Van Name ; D. carnulentum Ritter \& Forsyth et $D$. pardum Tokioka. Didemnum vestum Kott, 2004 est considéré comme un synonyme plus récent de D. vexillum Kott, 2002 (Lambert, 2009).

L'espèce est originaire du Japon (Lambert, 2009).

Cette espèce, invasive, est dangereuse pour l'équilibre écologique du milieu où elle se développe. Non seulement elle concurrence les suspensivores, mais en plus elle étouffe les organismes qu'elle recouvre. De plus c'est une salissure qui peut développer une biomasse importante, et constituer une gêne à la navigation (Boudouresque, 2005; Minchin \& Sides, 2006).

\section{LES ESPÈCES INTRODUITES DU PORT D'ANTIFER (MANCHE ORIENTALE)}

Le port d'Antifer (Saint-JouinBruneval, Seine-Maritime) a été exploré entre 2007 et 2011 au cours de 18 plongées dans le port pétrolier (dont deux de nuit) et deux dans le port de service. C'est dire que cette étude, faute d'un recul suffisant, ne permet pas de dater l'arrivée des espèces introduites, ni de préciser sérieusement l'évolution des populations. La liste des 237 taxons animaux qui ont été reconnus en plongée sur ce site est donnée en annexe 4. Cette bonne richesse spécifique s'explique en partie par la variété des milieux explorés: fond sédimentaire vaseux, sablo-vaseux ou sableux, enrochements à petits blocs, enrochements à très gros blocs avec de grandes cavités, parois verticales des piliers des appontements, le tout entre le niveau des hautes mers et $29 \mathrm{~m}$. Les 13 espèces introduites que nous y avons repérées représentent donc une faible proportion de la biodiversité totale $(5,5 \%)$ et se répartissent entre 6 espèces anciennement introduites (< 1970), considérées comme naturalisées et 7 espèces d'introduction plus récente (> 1970) (Tab. II).

Toutes, sauf Megabalanus tintinnabulum (Linnaeus) vivent aussi dans le port du Havre. La plupart se retrouve également sur la côte autour du port d'Antifer. Pour certaines (Fenestrulina delicia, Perophora japonica, Megabalanus tintinnabulum), c'est le manque de données qui nous interdit de conclure ; leur absence sur le littoral n'est pas démontrée. Le reste de la faune, dont la liste est donnée en annexe 3 , est très identique à ce que l'on observe sur la côte, en intertidal et infratidal proche, autour du port d'Antifer.

Le port d'Antifer n'a pas les caractères d'un bassin paralique, ce qui est cohérent avec l'excellent renouvellement de l'eau. Tout au plus peut-on noter que le port de service aurait un cachet paralique un peu plus accusé que le port pétrolier ; il pourrait relever de la zone I-II. Aucune des espèces introduites recensées dans le port d'Antifer ne présente de caractère invasif. Aucune de ces espèces n'appelle de commentaires particuliers autres que ceux qui leur ont été consacrés dans les paragraphes 4 et 5 . 
Tableau II. Liste des espèces animales introduites identifiées dans le port pétrolier d'Antifer (SeineMaritime), 2007-2011.

Introduction récente : espèce arrivée en Europe après 1970. Naturalisée : espèce arrivée en Europe avant 1970. Aucune des espèces recensées n'a montré un caractère invasif dans le port d'Antifer. Table II. List of the alien animal species identified in the port of Antifer (Seine-Maritime), 2007-2011. Recent introduction: species introduced in Europe after 1970. Naturalized: species introduced in Europe before 1970. None of these species are invasive in the port of Antifer.

\begin{tabular}{|c|c|c|}
\hline Espèces & Statut & Caractère invasif \\
\hline \multicolumn{3}{|l|}{ Cténaires } \\
\hline $\begin{array}{l}\text { Mnemiopsis leidyi } \\
\text { (A. Agassiz) }\end{array}$ & Introduction récente & Non invasive dans le port d'Antifer \\
\hline \multicolumn{3}{|l|}{ Mollusques } \\
\hline Ensis directus Conrad & Introduction récente & Non invasive dans le port d'Antifer \\
\hline $\begin{array}{l}\text { Crepidula fornicata } \\
\text { (Linnaeus) }\end{array}$ & Naturalisée & Non invasive dans le port d'Antifer \\
\hline \multicolumn{3}{|l|}{ Crustacés } \\
\hline $\begin{array}{l}\text { Austrominius modestus } \\
\text { (Darwin) }\end{array}$ & Naturalisée & Non invasive dans le port d'Antifer \\
\hline Balanus improvisus Darwin & $\begin{array}{l}\text { Cryptogénique? } \\
\text { Naturalisée }\end{array}$ & Non invasive dans le port d'Antifer \\
\hline $\begin{array}{l}\text { Megabalanus tintinnabulum } \\
\text { (Linnaeus) }\end{array}$ & $\begin{array}{l}\text { Cryptogénique? } \\
\text { Naturalisée? }\end{array}$ & Non invasive dans le port d'Antifer \\
\hline $\begin{array}{l}\text { Hemigrapsus sanguineus } \\
\text { de Haan }\end{array}$ & Introduction récente & Non invasive dans le port d'Antifer \\
\hline \multicolumn{3}{|l|}{ Bryozoaires } \\
\hline Bugula neritina (Linnaeus) & Naturalisée & Non invasive dans le port d'Antifer \\
\hline Bugula stolonifera Ryland & Naturalisée & Non invasive dans le port d'Antifer \\
\hline $\begin{array}{l}\text { Fenestrulina delicia Winston, } \\
\text { Hayward \& Craig }\end{array}$ & Introduction récente & Non invasive dans le port d'Antifer \\
\hline \multicolumn{3}{|l|}{ Ascidies } \\
\hline Botrylloides violaceus Oka & Introduction récente & Non invasive dans le port d'Antifer \\
\hline Perophora japonica Oka & Introduction récente & Non invasive dans le port d'Antifer \\
\hline Styela clava Herdmann & Naturalisée & Non invasive dans le port d'Antifer \\
\hline
\end{tabular}




\section{LES ESPÈCES INTRODUITES DU PORT DE ROUEN (BASSIN DE LA SEINE) (FIG. 18)}

Le port de Rouen (Seine-Maritime) a été exploré entre 2010 et 2012 au cours de 9 plongées cumulant près de 47 heures d'immersion. À part une plongée en 2010 dans le bassin aux Bois, dont le fond sédimentaire s'est avéré extrêmement pollué par des hydrocarbures, toutes les explorations ont eu lieu dans le bassin SaintGervais, autour du ponton de plaisance (5 plongées), et autour du confluent avec le Cailly (3 plongées), au printemps ou en automne. C'est dire que les résultats présentés ci-dessous doivent être considérés comme partiels et provisoires ; ils ne permettent pas non seulement de dater l'arrivée d'espèces introduites (sauf Compsopogon aeruginosus (J. Agardh) Kütz., (Fig. 18), cas particulier), mais encore de préciser sérieusement l'évolution des populations, introduites ou non. Une enquête auprès de pêcheurs a permis de dresser une liste complémentaire de poissons présents dans le port de Rouen, dont la plupart sont difficilement observables en plongée. Une liste complète des taxons recensés dans le port de Rouen est donnée en annexe 5, le tableau III dresse la liste commentée des espèces introduites présentes en 2010-2012 dans le port de Rouen (Bassin Saint-Gervais).

Les taxons reconnus dans le port de Rouen se répartissent ainsi (annexe 5) :

- taxons végétaux (procaryotes, algues, bryophytes, végétaux supérieurs) : 41 dont 3 introduits ;

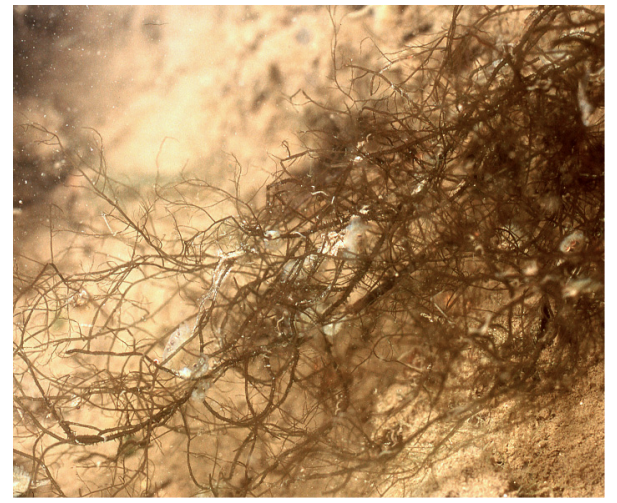

Fig. 18. Compsopogon aeruginosus (J. Agardh) Kütz. Bassin Saint-Gervais, port de Rouen, 23.09.2012.

Fig. 18. Compsopogon aeruginosus (J. Agardh) Kütz. Saint-Gervais basin, port of Rouen, 23.09.2012.

- taxons animaux : 50 [58 avec les poissons de l'enquête] dont 9 [11] introduits ;

- taxons totaux : 90 [99] dont 12 [14] introduits.

Les taxons introduits représentent donc environ $14 \%$ des taxons totaux. Ils se répartissent ainsi : algues : 1 ; phanérogames : 2 ; mollusques : 3 ; crustacés : 5 ; bryozoaires : 1 ; poissons : 2 . Nous prenons ici en compte toutes les espèces considérées comme introduites en France par les auteurs, quelle que soit l'ancienneté de leur introduction (i.e. espèces naturalisées comprises) et quel que soit leur statut, invasif ou non.

À part l'introduction très récente et probablement ponctuelle d'une algue rouge, attribuée à un rejet d'aquarium (Breton, 2013), et dont la naturalisation future semble peu vraisemblable, toutes les espèces introduites du port 
Tableau III. Espèces introduites recensées dans le port de Rouen (Seine-Maritime) en 2010-2012. Table III. Introduced species of the port of Rouen (Seine-Maritime) in 2010-2012.

(1) : Breton, 2013. (2) : Hudin et al. (2010). (3) : Mouthon (1981), Vincent \& Brancotte (2000, 2002).

(4) : Beisel \& Devin (2006), Bouquerel (2008). (5) : Ludovic Huriez (com. pers.). (6) : Beisel (2007).

(7) : Schlumberger et al. (2001).

\begin{tabular}{|c|c|c|c|}
\hline Espèces & Origine & Statut en France & Remarques \\
\hline \multicolumn{4}{|l|}{ Rhodophycées } \\
\hline $\begin{array}{l}\text { Compsopogon } \\
\text { aeruginosus } \\
\text { (J.Agardh) Kütz. } \\
\text { (Fig. 18) }\end{array}$ & Circumtropicale & Introduite en 2012 (1) & $\begin{array}{l}\text { Provient vraisemblablement } \\
\text { d'un rejet d'aquarium }\end{array}$ \\
\hline \multicolumn{4}{|l|}{ Phanérogames } \\
\hline $\begin{array}{l}\text { Elodea nuttalii } \\
\text { (Planch) H. St } \\
\text { John }\end{array}$ & Amérique du Nord & Naturalisée (2) & $\begin{array}{l}\text { Peuabondante, noninvasive } \\
\text { dans le port de Rouen }\end{array}$ \\
\hline $\begin{array}{l}\text { Vallisneria } \\
\text { spiralis } \\
\text { Linnaeus }\end{array}$ & $\begin{array}{l}\text { Circumtropicale- } \\
\text { subtropicale }\end{array}$ & Naturalisée & $\begin{array}{l}\text { Première introduction en } \\
\text { Bourgogne-Savoie-Rhône, } \\
\text { avait disparu de la Seine } \\
\text { depuis les années 70', et y } \\
\text { avait fait une réapparition } \\
\text { récente (8) }\end{array}$ \\
\hline \multicolumn{4}{|l|}{ Mollusques } \\
\hline $\begin{array}{l}\text { Dreissena } \\
\text { polymorpha } \\
\text { (Pallas) (Fig. 19) }\end{array}$ & Pontocaspienne & \begin{tabular}{|l|} 
Apparue au milieu du \\
$19^{\mathrm{e}}$ siècle. \\
Naturalisée. \\
Localement invasive
\end{tabular} & $\begin{array}{l}\text { Possiblement invasive dans } \\
\text { le port de Rouen : les popu- } \\
\text { lations sont très denses. }\end{array}$ \\
\hline $\begin{array}{l}\text { Corbicula } \\
\text { fluminea } \\
\text { (Rafinesque) }\end{array}$ & $\begin{array}{l}\text { Asie tropicale } \\
\text { et subtropicale }\end{array}$ & $\begin{array}{l}\text { Introduction récente } \\
\text { (1980). Invasive (3) }\end{array}$ & $\begin{array}{l}\text { Statut taxinomique discuté : } \\
1,2 \text { ou } 3 \text { espèces introduites } \\
\text { distinctes en France? Non } \\
\text { invasive dans le port de } \\
\text { Rouen }\end{array}$ \\
\hline $\begin{array}{l}\text { Menetus } \\
\text { dilatatus } \\
\text { (Gould) }\end{array}$ & Amérique du Nord & $\begin{array}{l}\text { Introduction récente. } \\
\text { Expansion rapide }\end{array}$ & $\begin{array}{l}\text { Non invasive dans le port de } \\
\text { Rouen }\end{array}$ \\
\hline \multicolumn{4}{|l|}{ Crustacés } \\
\hline $\begin{array}{l}\text { Chelicorophium } \\
\text { curvispinum } \\
\text { (G. O. Sars) }\end{array}$ & Pontocaspienne & $\begin{array}{l}\text { Introduction récente } \\
\text { (1994), proliférante. }\end{array}$ & Rare dans le port de Rouen \\
\hline $\begin{array}{l}\text { Atyaephyra } \\
\text { desmaresti } \\
\text { (Millet) }\end{array}$ & $\begin{array}{l}\text { Afrique du Nord } \\
\text { et Europe du Sud }\end{array}$ & $\begin{array}{l}\text { Introduction } \\
\text { ancienne, naturalisée }\end{array}$ & $\begin{array}{l}\text { Introduction ou bien exten- } \\
\text { sion vers le nord de l'aire de } \\
\text { répartition? Rare dans le } \\
\text { port de Rouen (1 observa- } \\
\text { tion). Espèce d'eauxpropres } \\
\text { et bien oxygénée (5), mais } \\
\text { euryhaline et eurytherme (6) }\end{array}$ \\
\hline
\end{tabular}


Tableau III. Suite

Table III. Continued.

\begin{tabular}{|c|c|c|c|}
\hline Espèces & Origine & Statut en France & Remarques \\
\hline $\begin{array}{l}\text { Orconectes } \\
\text { limosus } \\
\text { (Rafinesque) } \\
\text { (Fig. 20) }\end{array}$ & Amérique du Nord & $\begin{array}{l}\text { Introduite (début } \\
20^{e} \text { siècle). Invasive. A } \\
\text { supplanté l'écrevisse } \\
\text { indigène. }\end{array}$ & $\begin{array}{l}\text { Fréquente dans le port de } \\
\text { Rouen }\end{array}$ \\
\hline \multicolumn{4}{|l|}{ Bryozoaires } \\
\hline $\begin{array}{l}\text { Pectinatella } \\
\text { magnifica (Leidy) }\end{array}$ & Amérique du Nord & $\begin{array}{l}\text { Introduction ancienne } \\
\text { en Europe. Naturalisée }\end{array}$ & $\begin{array}{l}\text { Reconnue à Rouen seule- } \\
\text { ment par ses statoblastes }\end{array}$ \\
\hline \multicolumn{4}{|l|}{ Poissons } \\
\hline $\begin{array}{l}\text { Ameiurus melas } \\
\text { (Rafinesque) }\end{array}$ & Amérique du Nord & Naturalisé & $\begin{array}{l}\text { Donnée issue de l'enquête } \\
\text { auprès des pêcheurs }\end{array}$ \\
\hline $\begin{array}{l}\text { Silurus glanis } \\
\text { Linnaeus }\end{array}$ & $\begin{array}{l}\text { Pontocaspienne } \\
\text { et balte }\end{array}$ & $\begin{array}{l}\text { Naturalisée, } \\
\text { invasive (7) }\end{array}$ & $\begin{array}{l}\text { Donnée issue de l'enquête } \\
\text { auprès des pêcheurs }\end{array}$ \\
\hline
\end{tabular}

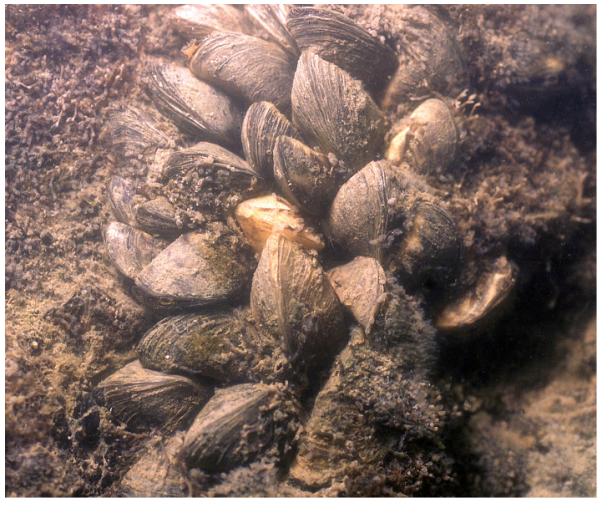

Fig. 19. Dreissena polymorpha (Pallas). Bassin Saint-Gervais, port de Rouen, 11.06.2011.

Fig. 19. Dreissena polymorpha (Pallas). SaintGervais basin, port of Rouen, 11.06.2011.

de Rouen sont connues dans le reste du réseau hydrographique de France métropolitaine (p. ex. Bouquerel, 2008 ; Hudin et al., 2010).

\section{DISCUSSION ET CONCLUSIONS}

Les espèces animales provenant d'une introduction ancienne présentes

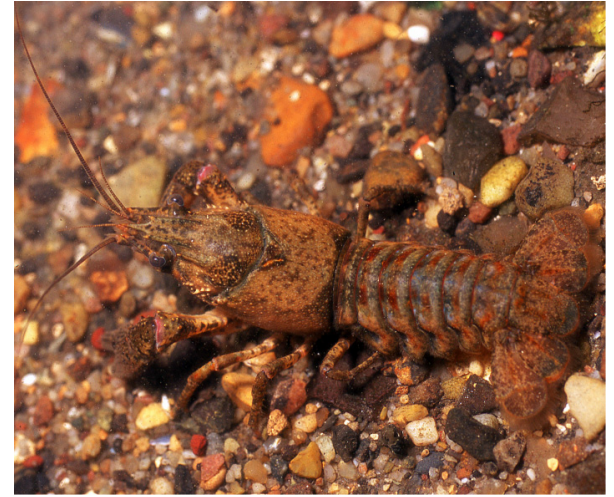

Fig. 20. Orconectes limosus (Rafinesque). Bassin Saint-Gervais, port de Rouen, 23.09.2012.

Fig. 20. Orconectes limosus (Rafinesque). Saint-Gervais basin, port of Rouen, 23.09.2012.

dans le port du Havre proviennent aux deux tiers du Pacifique $\mathrm{N}$ ou NW, en particulier du Japon, ou bien de la côte E de l'Amérique du Nord. Si une majorité d'espèces animales d'introduction récente a la même origine, la provenance géographique des autres se diversifie. Cela est lié à la diversification du trafic maritime à partir des années 60. En effet, il est reconnu 
depuis longtemps (par exemple Carlton, 1985 ; Boudouresque, 2005 ; Leppäkoski, 2007) que le trafic maritime est le principal facteur de dispersion des espèces, transportées le plus souvent sous forme larvaire ou forme de résistance dans les eaux de ballast des navires.

Dans le port de Rouen, l'origine géographique des espèces introduites animales et végétales est plus varié : Amérique du Nord (5), pontocaspienne (4), circumtropicale (3) et Europe du SW - Afrique du Nord (2). Ces différentes origines correspondent à la variété des modes et des vecteurs d'introduction. Les espèces d'origine pontocaspienne ont gagné l'Europe du Nord-Ouest (et parfois du Nord) par les fleuves et les canaux. Les espèces nord-américaines et tropicales relèvent plus de l'action de l'homme, par exemple une introduction volontaire « de repeuplement» (Orconectes limosus: Fig. 20) ou liée aux besoins ou aux développements de l'aquariophilie (Compsopogon aeruginosus: Fig. 19).

La proportion d'espèces animales introduites représente :

- au Havre 9,9 \% de la richesse spécifique animale totale [introductions anciennes $5,2 \%$; introductions récentes 4,7\%] ;

- à Antifer 5,5\% de la faune recensée se répartissant à parts à peu près égales entre introductions anciennes et récentes ;

- à Rouen $14 \%$ de la flore et de la faune totales, se répartissant à parts à peu près égales entre introductions anciennes et récentes ;
Au-delà des proportions d'espèces introduites et de leurs origines géographiques, la comparaison entre les peuplements et les espèces introduites des trois ports de Seine-Maritime étudiés, les ports du Havre et d'Antifer en eau de mer et le port de Rouen en eau douce, met en évidence une originalité du port du Havre. Les peuplements d'espèces indigènes et introduites du port de Rouen sont ceux que l'on rencontrerait en n'importe quel point du réseau hydrographique de France métropolitaine, dans un plan d'eau lentique non salé dépendant du cours aval d'un fleuve ou d'une rivière. II n'y a pas de taxon original, la seule originalité du port de Rouen étant la présence d'une zone de balancement des marées. De même, les peuplements animaux d'espèces indigènes et introduites du port d'Antifer sont ceux que l'on peut observer, à profondeur équivalente, sur le littoral alentour.

Dans plusieurs cas au contraire, le port du Havre a enregistré l'arrivée d'une nouvelle espèce introduite avant qu'elle ne se répande de manière généralisée en Manche orientale. Ce fut le cas de Styela clava, Mnemiopsis leidyi, Caprella mutica, Hemigrapsus takanoi, Hemigrapsus sanguineus, Tricellaria inopinata, Corella eumyota, Botrylloides violaceus et Didemnum vexillum. Parfois l'espèce immigrante reste confinée au port du Havre, par exemple Hydroides ezoensis.

Nous pouvons, semble-t-il, invoquer trois raisons. D'une part, la présence sur place d'une équipe de plongeurs scientifiques capables de reconnaître l'arrivée d'une espèce non encore repérée a certainement joué. 
Ensuite, le port est, par définition, un lieu d'étape des navires, vecteurs majeurs du transport d'espèces, donc un site d'accueil privilégié de ces espèces (Carlton, 1985 ; Boudouresque, 2005 ; Leppäkoski, 2007). Enfin, le milieu d'accueil, quoique fortement anthropisé, relève du domaine paralique (sensu Guélorget \& Perthuisot, 1983). Les grandes fluctuations démographiques des espèces présentes, fluctuations typiques du domaine paralique, ouvrent régulièrement des niches écologiques que les nouveaux arrivants, espèces opportunistes en général, peuvent coloniser. D'autant plus que la productivité primaire en domaine paralique est forte: les ressources trophiques pour les nouveaux arrivants, quelles que soient leurs places dans les chaînes alimentaires, sont disponibles. C'est d'ailleurs ces propriétés que Boudouresque (2005, p. 40-42) invoque - sans toutefois évoquer le caractère paralique de ces milieux - pour expliquer le succès des invasions.

La combinaison du caractère opportuniste de l'immigrant et des opportunités apportées par le caractère paralique des plans d'eau colonisés fait que cette colonisation se fait parfois de manière invasive. Ce fut le cas de Mnemiopsis leidyi, Caprella mutica, Hydroides ezoensis et Didemnum vexillum. Ce caractère invasif entraîne parfois une augmentation spectaculaire de la biomasse (Didemnum vexillum, Mnemiopsis leidyı) ; il entraîne aussi l'éviction d'espèces indigènes, phénomène moins spectaculaire dans l'instant, mais aux conséquences probablement plus lourdes.
Le fait que le domaine paralique « répare » rapidement les conséquences d'un désordre écologique profond (comme les épisodes de dystrophie et d'anoxie, par exemple les “ malaïgues » de l'étang de Thau) aura deux conséquences distinctes. D'une part, certaines populations peuvent « exploser » (Ficopomatus enigmaticus) dans les niches écologiques laissées libres par les populations décimées. D'autre part, c'est probablement par un mécanisme comparable que beaucoup d'invasions connaissent une phase initiale «explosive » avant de régresser, par exemple Didemnum vexillum. La régression n'est pas toujours la règle, par exemple Hydroides ezoensis ou Caprella mutica. II faut souligner que certaines introductions anciennes ont été détectées régionalement en milieu paralique: Ficopomatus enigmaticus dans le Canal de Caen à la mer, ou encore Rithropanopeus harrissii dans le Canal de Tancarville.

Le port du Havre est donc un plan d'eau « sentinelle » pour la détection d'espèces introduites, voire invasives, parce qu'il appartient au domaine paralique (Breton, 2005), par opposition au port d'Antifer, plan d'eau identique du point de vue bionomique au littoral qui l'entoure ou au port de Rouen, plan d'eau dont le peuplement est semblable à celui du réseau hydrographique auquel il appartient.

\section{REMERCIEMENTS}

Mes remerciements vont tout d'abord à l'équipe de l'association Port Vivant. II m'est impossible de citer ici 
tous mes compagnons de plongée qui ont peu ou prou contribué à améliorer nos connaissances sur les populations des ports. Que chacun accepte mes remerciements et en particulier Denis Corthésy sans qui Port Vivant n'existerait pas et Daniel Ingratta qui a permis la publication d'une de ses excellentes photos. Mes remerciements à ceux qui nous ont accompagnés dans nos recherches, Chloé Dancie, Aurélien Berno (Cellule de Suivi du Littoral Normand) et Thierry Ruellet (GEMEL), ainsi que le GIP Seine Aval. Un grand merci à Yves Müller et Marc Damerval qui nous ont aidés dans l'identification de bivalves, à Françoise Monniot qui a toujours accepté d'étudier et d'identifier nos récoltes d'ascidies et à Patrick Bertrand qui nous a renseignés efficacement sur l'histoire du port du Havre. Enfin, nous ne saurions oublier de remercier le Centre de production thermique EDF du Havre pour l'intérêt qu'il porte à nos travaux ; la Capitainerie du Grand Port Maritime du Havre qui a toujours examiné avec une rigoureuse attention nos demandes de plongées, le Service Environnement du GPMH qui a témoigné régulièrement de l'intérêt pour nos recherches, et le Grand Port Maritime de Rouen, Service Environnement et Capitainerie, pour leur accueil et leur intérêt.

\section{RÉFÉRENCES BIBLIOGRAPHIQUES}

Antajan E., Raud T., Brylinski J.-M., Hoffmann S., Breton G., Cornille V., Delegrange A. \& Vincent D. First records of Mnemiopsis leidyi A. Agassiz 1865 along the English Channel and the North Sea French coasts. Aquatic Invasions, sous presse.
Appeltans W., Bouchet P., Boxshall G.A., De Broyer C., de Voogd N.J., Gordon D.P., Hoeksema B.W., Horton T., Kennedy M., Mees J., Poore G.C.B., Read G, Stöhr S., Walter T.C. \& Costello M.J. (Eds), 2012. World Register of Marine Species. Accessed at http:// www.marinespecies.org on 2013-07-07.

Arenas F., Bishop J.D.D., Carlton J.T., Dyrynda P.J., Farnham W.F., Gonzales D.J., Jacobs M.W., Lamberts C., Lambert G., Nielsen S.E., Pederson J.A., Porter J.S., Ward S. \& Wood C.A., 2006. Alien species and other notable records from a rapid assessment survey of marinas on the south coast of England. Journal of the Marine Biological Association of the United Kingdom 86 : 1329-1337.

Asakura A. \& Watanabe S., 2005. Hemigrapsus takanoi, new species, a sibling species of the common Japanese intertidal crab $H$. penicillatus (Decapoda: Brachyura: Grapsoidea). Journal of Crustacean Biology 25 : 279-292.

Bay-Nouailhat A., 2007. Description de Perophora japonica, [En ligne] http:// www.mer-littoral.org/32/perophorajaponica.php, consultée le 23 juin 2013.

Beisel J.N., 2007. Atyaephyra desmaresti in Invasions biologiques. [En ligne] http:// www.invabio.univ-metz.fr/

z_dikerogammarus_villosus.ht [consulté le 7 juillet 2013].

Beisel J.N. \& Devin S., 2006. Dikerogammarus villosus in Invasions biologiques. [En ligne] http://www.invabio.univ-metz.fr/ z_atyaephyra_desmaresti.ht [consulté le 7 juillet 2013].

Bishop J., 2005. Perophora japonica. A sea squirt. Marine Life Information Network: Biology and Sensitivity Key Information Sub-programme [on-line]. Marine Biological Association of the United Kingdom, Plymouth [cited 23/06/ 2013]. Available from: http://www.marlin.ac.uk/speciesinformation.php?species $\mid \mathrm{D}=4074$. 
Blanchard M., 1995. Origine et état de la population de Crepidula fornicata (Gastropoda Prosobranchia), sur le littoral français. Haliotis 24 : 75-86.

Blanchard M., 1997. Spread of the sliperlimpet Crepidula fornicata (L. 1758) in Europe. Current state and consequences. Scientia marina 61, Suppl. 2 : 109-118.

Boudouresque C.F., 2005. Les espèces introduites et invasives en milieu marin. Deuxième édition. Gis Posidonie publ. Marseilles [sic] : $152 \mathrm{p}$. [version mise à jour octobre 2006 : 170 p.]

Bouquerel J., 2008. Les canaux : des milieux privilégiés pour les macroinvertébrés invasifs. Étude de la région Nord-Pas-de-Calais. Rapport d'études. Agence de l'eau Artois-Picardie et Université des Sciences et technologies de Lille 1, Laboratoire d'Écologie numérique et d'Écotoxicologie, $81 \mathrm{p}$.

Breton G., 1981a. Capture du «crabe bleu " Callinectes sapidus (Rathbun, 1896) dans le port du Havre. In compte rendu des réunions mensuelles. Bulletin trimestriel de la Société géologique de Normandie et des Amis du Muséum du Havre 68, 4 : 8-9.

Breton G., 1981b. Observations sur l'écologie et les peuplements des bassins du port du Havre (France). Bulletin trimestriel de la Société géologique de Normandie et des Amis du Muséum du Havre 68, 4 : 54-54.

Breton G., 2005. Le port du Havre (Manche orientale, France) et ses peuplements : un exemple de domaine paralique en climat tempéré. Bulletin de la Société Zoologique de France 130, 4 : 381-423.

Breton G., 2013. Introduction de l'algue rouge Compsopogon aeruginosus (J. Agardh) Kützing dans le port de Rouen (Normandie, France). Hydroécologie Appliquée, DOI : 10.1051/hydro/2013052 : 1-8.
Breton G. Observation du Sar commun atlantique Diplodus sargus cadenati de la Paz, Bauchot \& Daget, 1974 dans le port du Havre. Bulletin de la Société linnéenne de Normandie, Caen, sous presse.

Breton G. \& Dupont W., 1978. Styela clava Herdmann, Ascidie nouvelle pour les côtes de la baie de Seine, abonde dans le port du Havre (76). Bulletin trimestriel de la Société géologique de Normandie et des Amis du Muséum du Havre 65, $2: 51$.

Breton G. \& Hondt J.-L., d' 2005. Tricellaria inopinata d'Hondt et Occhipinti Ambrogi, 1985 (Bryozoa : Cheilostomatida) dans le port du Havre (Manche orientale). Bulletin trimestriel de la Société géologique de Normandie et des Amis du Muséum du Havre 91, 2 : 67-72.

Breton G. \& Huriez L., 2010. Les crépidules Crepidula fornicata (Linnaeus, 1758) peuvent temporairement redevenir brouteuses à l'âge adulte. Bulletin de la Société linnéenne de Normandie 120 : 98-104.

Breton G., Girard A. \& Lagardère J.-P., 1996. Espèces animales benthiques des bassins du port du Havre (Normandie, France), rares, peu connues ou nouvelles pour la région. Bulletin trimestriel de la Société géologique de Normandie et des Amis du Muséum du Havre 82, 3 : 7-28.

Breton G., Faasse M., Noël P. \& Vincent T., 2002. A new alien crab in Europe: Hemigrapsus sanguineus (Decapoda: Brachyura: Grapsidae). Journal of Crustacean Biology 22, 1 : 184-189.

Carlton J.T., 1985. Transoceanic and interoceanic dispersal of coastal marine organisms: the biology of ballast water. Oceanography and Marine Biology 23 : 313-371. 
Cook E.J., Jahnke M., Kerckof F., Minchin D., Faasse M. \& Boos K., 2007. European expansion of the introduced amphipod Caprella mutica Schurin 1935. Aquatic Invasions 2, $4:$ : 411-421.

Crisp D.T., 1958. The spread of Elminius modestus Darwin in the nort-west Europe. Journal of the Marine Biological Association of the United Kingdom 37 : 483-520.

Dauvin J.C. (coord.), 1997. Les biocénoses marines et littorales françaises des côtes Atlantique, Manche, Mer du Nord. IEBG, Paris, $376 \mathrm{p}$.

De Blauwe H., 2009. Mosdiertjes van de Zuidelijke Bocht van de Noordzee. Determinatiewerk voor België en Nederland. Uitgave Vlaams Institut voor de Zee, Oostende : 464 p.

Dégremont C. \& Levêque C., 2012. La Seine en Normandie. GIP Seine-Aval, Rouen : $229 \mathrm{p}$.

Dewarumez J.-M., Gevaert F., Massé C., Foveau A. \& Grulois D., 2011. Les espèces marines animales et végétales introduites dans le bassin Artois-Picardie. Agence de l'eau Artois-Picardie, LOG, et UMR CNRS 8187, Wimereux : $140 \mathrm{p}$.

Eno N.C., Clarck R.A. \& Sanderson W.G. (Eds), 1997. Non-native marine species in British waters: a review and directory. Joint Nature Conservation Committee, Peterborough : $151 \mathrm{p}$.

Galil B.S., Clark P.F. \& Carlton J.T. (Eds), 2011. In the wrong place - Alien marine crustacean: distribution, Biology and impacts. Series Invading nature, Springer series in Invasion Ecology 6: $716 \mathrm{p}$.

Gollash S., 1999. The Asian decapod Hemigrapsus penicillatus (de Haan, 1835) (Grapsidae, Decapoda) introduced in European waters: status quo and future perspectives. Helgoländer Meeresuntersuchungen 52 : 359-366.

Goulletquer P., Bachelet G., Sauriau P.G. \& Noël P., 2002. Open Atlantic coast of Europe: a century of introduced species into French waters. In Invasive aquatic species of Europe, Leppäkoski, Gollash \& Olnin (Eds), Kluver Academic Publishers, Dordrech : Annexe 1 [non paginé].

Gruet Y. \& Baudet J., 1977. Les introductions d'invertébrés marins. In Les biocénoses marines et littorales françaises des côtes Atlantique, Manche, Mer du Nord (J.-C. Dauvin (coord.). IEBG, Paris : 242-250.

Gruet Y., Héral M. \& Robert J.-M., 1976. Premières observations sur l'introcustion de la faune associée au naissain d'huitres japonaises Crassostrea gigas (Thunberg), importé sur la côte atlantique française. Cahiers de Biologie Marine 17 : 173-184.

Guélorget O. \& Perthuisot J.-P., 1983. Le domaine paralique, expressions géologiques, biologiques et économiques du confinement. Travaux du Laboratoire de Géologie 16 : $136 \mathrm{p}$.

Hondt J.-L. d' \& Occhipinti Ambrogi A., 1985. Tricellaria inopinata, n. sp., un nouveau Bryozoaire Cheilostome de la faune méditerranéenne. Marine Eco$\log y 6,1: 35-46$.

Hondt J.-L. d', Occhipinti Ambrogi A. \& Goyffon M., 2004. Étude comparée du polymorphisme électrophorétique chez deux familles de Bryozoaires Cellularines: les Candidae et les Bugulidae. Bulletin de la Société Zoologique de France 138, 3 : 161-183.

Hudin S., et al. ${ }^{*}$, 2010. Guide d'identification des plantes exotiques envahissant les milieux aquatiques et les berges du bassin Loire - Bretagne, Fédération des conservatoires d'espaces naturels, $45 \mathrm{p}$. [ ${ }^{\star}$ référence bibliographique recommandée par les auteurs].

Lambert G., 2004. The south temperate and Antarctic ascidian Corella eumyota reported in two harbours in north-western France. Journal of the Marine Biological Association of the United Kingdom 84 : 239-241. 
Lambert G., 2009. Adventures of a sea squirt sleuth: unravelling the identity of Didemnum vexillum, a global ascidian invader. Aquatic Invasions 4, 1 : 5-28.

Lapègue S., Boudry P. \& Goulletquer P., 2006. Pacific cupped oyster - Crassostrea gigas. In Genetic effects of domestication, culture and breeding of fish and shellfish, and their impacts on wild populations, D. Crosetti, S. Lapègue, I. Olesen, T. Svaasand (Eds), GENIMPACT project: Evaluation of genetic impact of aquaculture activities on native populations. A European network. WP1 workshop "Genetics of domestication, breeding and enhancement of performance of fish and shellfish", Viterbo, Italy, 12-17th June, 2006, 6 p. http://genimpact.imr.no/

Lavesque N., Bachelet G., Béguer M., Girardin M., Lepage M., Blanchet H., Sorbe J.-C., Modéran J., Sauriau P.-G. \& Aubry I., 2010. Recent expansion of the oriental shrimp Palaemon macrodactylus (Crustacea : Decapoda) on the western coasts of France. Aquatic Invasions 5, Suppl. : 103-108.

Ledoyer M., 1989. Les Mysidacées (Crustacea) des grottes sous-marines obscures de Méditerranée nord-occidentale et du proche Atlantique (Portugal et Madère). Marine Nature 2, 1 : 39-62.

Leppäkoski E., 2007. Shipping, the most important vector of aquatic alien species. In Alien species, Korpimaki E. \& Nordström M. (conv.) 5th Environment symposium of the Maj and Tor Nessling Foundation, 18-19.01.2007, Arken, Turku, Finland : 21.

Le Duff M., Breton G. \& Ar Gall E., 2008. Caulacanthus ustulatus, la petite algue rouge qui monte, qui monte... Penn ar bed 202 : 14-20.

Manuel R.L., 1988. British Anthozoa (Coelenterata: Octocorallia \& Hexacorallia). Synopses of the British Fauna (New Series) 18 (rev.) : $241 \mathrm{p}$.
Minchin D. \& Sides E., 2006. Appearance of a cryptogenic tunicate, a Didemnum sp. fouling marina pontoons and leisure crafts in Ireland. Aquatic Invasions 1, 3 : 143-147.

Monniot C. \& Monniot F., 1985. Apparition de l'ascidie Perophora japonica sur les côtes et dans les ports de la Manche. Compte rendu des séances de la Société de Biogéographie 61 : 111-116.

Monniot F. The genus Corella (Ascidiacea, Phlebobranchia, Corellidae) in the southern hemisphere with description of a new species. Zootaxa, soumis.

Mouthon J., 1981. Sur la présence en France et au Portugal de Corbicula (Bivalvia, Corbiculidae) originaire d'Asie. Basteria 45 : 109-116.

Nijland R. \& Beekman J., 2000. Hemigrapsus penicillatus (de Haan, 1835) waargenomen in Nederland. Het Zeepaard, Leiden, 60, 3 : 169-171.

Nishikawa T., Bisop J.D.D. \& Sommerfeldt D., 2000. Occurrence of the alien ascidian Perophora japonica at Plymouth. Journal of the Marine Biological Association of the United Kingdom 80 : 955-956.

Noël P.Y., 2011. Checklist of cryptogenic and alien Crustacea of the European Atlantic coast. In In the wrong place Alien marine crustacean: distribution, Biology and impacts, Galil et al., (Eds). Invading nature, Springer series in Invasion Ecology 6 : 345-375.

Noël P., Tardy E. \& Udekem d'Acoz C.d', 1977. Will the crab Hemigrapsus penicillatus invade the coasts of Europe? Comptes rendus de l'Académie des Sciences Paris, Sciences de la Vie 320 : 741-745.

Otero M., Cebrian E., Francour P., Galil B. \& Savini D., 2013. Surveilance des espèces envahissantes marines dans les aires marines protégées (AMP) méditerranéennes: guide pratique et stratégique à l'attention des gestionnaires. Medpan coll., IUCN : 136 p. 
Poppe G. \& Goto Y., 1993. European seashells, Vol. II (Bivalvia). Christa Hemmer, Wiesbaden : $221 \mathrm{p}$.

Schlumberger O., Sagglioco M. \& Proteau J.-P., 2001. Biogéographie du Silure Glane Silurus glanus : causes hydrographiques, climatiques et anthropiques. Bulletin français des Pêches et de la Pisciculture 357/360 : 533-547.

Southward A.J., 2008. Barnacles. Synopses of the British Fauna (New Series), Shrewsbury $57: 144 \mathrm{p}$.

Stefaniak L., Lambert G., Gittenberg A., Zhang H. \& Lin S., 2009. Genetic conspecificity of the worldwide populations of Didemnum vexillum Kott, 2002. Aquatic Invasions, 4, 1 : 29-44.

Thorp C.H., Pyne S. \& West S.A., 1987. Hydroides ezoensis Okuda, a fouling serpulid new to British coastal waters. Journal of Natural History 21: 863-877.

Udekem d'Acoz C. d' \& Faasse M., 2002. De huidige status van Hemigrapsus sanguineus (de Haan, 1835) en Hemigrapsus penicillatus (de Haan, 1835) in de noordlijke Atlantische Oceaan, in het bijzonder in Nederland, met opmerkingen over hun biologie (Crustacea, Decapoda, Brachyura). Het Zeepaard 62, 4 : 101-115.

Vincent T., 1987. Les captures de Callinectes sapidus (Rathbun, 1896) en baie de Seine entre 1975 et 1984 . Bulletin trimestriel de la Société géologique de Normandie et des Amis du Muséum du Havre 73, 4 : 13-15.

Vincent T., 1996. Le crabe chinois Eriocher sinensis H. Milne-Edwards, 1854 (Crustacea, Brachyura) en Seine-Maritime, France. Annales de l'Institut Océanographique, Paris, 72, 2 : 155-171.

Vincent T., 2001. Ichtyofaune des bassins du port du Havre (Normandie, France). Bulletin trimestriel de la Société géolo- gique de Normandie et des Amis du Muséum du Havre 87, 1 : 59-84.

Vincent T. \& Brancotte V., 2000. Le bivalve invasif Corbicula fluminea (Heterodonta, Sphaeriacea, Corbiculidae) dans le bassin hydrographique de la Seine (France), première prospection systématique et hypothèse sur la colonisation. Hydroécologie Appliquée 12, 1-2 : 147-158.

Vincent T. \& Brancotte V., 2002. Répartition actuelle et mode de progression de Corbicula spp. En France. Bulletin de la Société Zoologique de France, Paris, 127, $3:$ 241-252.

Vincent T. \& Breton G., 1999. Présence du crabe Hemigrapsus penicillatus (de Haan, 1835) dans les bassins du port du Havre (Normandie, France). Bulletin trimestriel de la Société géologique de Normandie et des Amis du Muséum du Havre 86, 1 : 19-23.

Vincent T. \& Noël P.-Y., 2002. Les Mysidacés et Décapodes capturés, collectés et observés entre 1978 et 2000 dans le port du Havre (Seine-Maritime, France). Bulletin trimestriel de la Société géologique de Normandie et des Amis du Muséum du Havre 87, 4 : 71-91.

Weerdt W.H. de, 1986. A systematic revision of the North-Eastern Atlantic shallow-water Haplosclerida (Porifera, Demospongiae), part II: Chalinidae. Beaufortia 36, 6 : 49-138.

Zibrowius H., 1978. Introduction du Polychète Serpulidae japonais Hydroides ezoensis sur la côte atlantique française, et remarques sur la propagation d'autres espèces de Serpulidae. Tethys 8, 2 : 141-150.

Zibrowius H. \& Thorp H.C., 1989. A review of alien serpulid and spirorbid Polychaetes in the British Isles. Cahiers de Biologie Marine 30 : 271-285. 
ANNEXE 1

Actualisation nomenclaturale des taxons animaux présents dans le port du Havre (Breton, 2005). Synonymies, nouvelles combinaisons, émendations orthographiques.

\begin{tabular}{|c|c|c|}
\hline Nom 2005 & Nom actuel & Remarques \\
\hline \multicolumn{3}{|l|}{ Porifera } \\
\hline Esperiopsis fucorum (Esper) & Amphilectus fucorum (Esper) & $\begin{array}{l}\text { Chang }^{\mathrm{t}} \\
\text { nom de genre }\end{array}$ \\
\hline Hymeniacidon perleve (Montagu) & Hymeniacidon perlevis (Montagu) & Accord \\
\hline \multicolumn{3}{|l|}{ Annelida Polychaeta } \\
\hline Aphrodite aculeata Linnaeus & Aphrodita aculeata Linnaeus & Orthographe \\
\hline Cirratulus filiformis Keferstein & Aphelochaeta filiformis (Keferstein) & $\begin{array}{l}\text { Chang }{ }^{t} \text { nom } \\
\text { de genre }\end{array}$ \\
\hline Hydroides norvegica Gunnerus & Hydroides norvegicus Gunnerus & Accord \\
\hline $\begin{array}{l}\text { Magalia permarmata Marion \& } \\
\text { Bobretzky ; Syllidia permarmata } \\
\text { Marion \& Bobretzky }\end{array}$ & Syllidia armata Quatrefages & Synonymie \\
\hline Neanthes succinea (Leuckart) & Alitta succinea (Leuckart) & $\begin{array}{l}\text { Chang }{ }^{\mathrm{t}} \text { nom } \\
\text { de genre }\end{array}$ \\
\hline Nereis longissima Johnston & Eunereis longissima Johnston & $\begin{array}{l}\text { Chang }^{t} \text { nom } \\
\text { de genre }\end{array}$ \\
\hline Nereis virens (Sars) & Alitta virens (Sars) & $\begin{array}{l}\text { Chang }^{\mathrm{t}} \text { nom } \\
\text { de genre }\end{array}$ \\
\hline $\begin{array}{l}\text { Ophiodromus flexuosus (Delle } \\
\text { Chiaje) }\end{array}$ & $\begin{array}{l}\text { Oxydromus flexuosus (Delle } \\
\text { Chiaje) }\end{array}$ & $\begin{array}{l}\text { Chang }^{\mathrm{t}} \text { nom } \\
\text { de genre }\end{array}$ \\
\hline $\begin{array}{l}\text { Pectinaria (Lagis) koreni } \\
\text { (Malmgren) }\end{array}$ & Lagis koreni (Malmgren) & $\begin{array}{l}\text { Chang }^{\mathrm{t}} \text { nom } \\
\text { de genre }\end{array}$ \\
\hline Pomatoceros lamarcki (Philippi) & $\begin{array}{l}\text { Spirobranchus lamarcki } \\
\text { (Quatrefages) }\end{array}$ & Synonymie \\
\hline Pomatoceros triqueter (Linnaeus) & Spirobranchus triqueter (Linnaeus) & $\begin{array}{l}\text { Chang }^{t} \text { nom } \\
\text { de genre }\end{array}$ \\
\hline Spirographis spallanzani Gmelin & Sabella spallanzani Gmelin & $\begin{array}{l}\text { Chang }^{\mathrm{t}} \text { nom } \\
\text { de genre }\end{array}$ \\
\hline $\begin{array}{l}\text { Streblespio schrubsholi } \\
\text { (Buchanan) }\end{array}$ & Streblespio shrubsholi (Buchanan) & Orthographe \\
\hline Tharyx marioni (de Saint-Joseph) & $\begin{array}{l}\text { Aphelochaeta marioni (de Saint- } \\
\text { Joseph) }\end{array}$ & $\begin{array}{l}\text { Chang }{ }^{t} \text { nom } \\
\text { de genre }\end{array}$ \\
\hline
\end{tabular}


ANNEXE 1

Suite.

\begin{tabular}{|c|c|c|}
\hline Nom 2005 & Nom actuel & Remarques \\
\hline Typosyllis cf. prolifera (Krohn) & Syllis cf. prolifera (Krohn) & $\begin{array}{l}\text { Chang }{ }^{t} \text { nom } \\
\text { de genre }\end{array}$ \\
\hline \multicolumn{3}{|l|}{ Crustacea } \\
\hline Elminius modestus Darwin & Austrominius modestus Darwin & $\begin{array}{l}\text { Changt nom } \\
\text { de genre }\end{array}$ \\
\hline Corophium acherusicum Costa & $\begin{array}{l}\text { Monocorophium acherusicum } \\
\text { Costa }\end{array}$ & $\begin{array}{l}\text { Chang }{ }^{t} \text { nom } \\
\text { de genre }\end{array}$ \\
\hline Chaetogammarus marinus (Leach) & Echinogammarus marinus (Leach) & $\begin{array}{l}\text { Chang }{ }^{t} \text { nom } \\
\text { de genre }\end{array}$ \\
\hline Polybius puber (Linnaeus) & Necora puber (Linnaeus) & $\begin{array}{l}\text { Chang }{ }^{t} \text { nom } \\
\text { de genre }\end{array}$ \\
\hline \multicolumn{3}{|l|}{ Mollusca } \\
\hline Venerupis pullastra (Montagu) & Venerupis corrugata (Gmelin) & Synonymie \\
\hline Venerupis decussatus (Linnaeus) & Venerupis decussata (Linnaeus) & Accord \\
\hline Hinia reticulata (Linnaeus) & Nassarius reticulatus (Linnaeus) & $\begin{array}{l}\text { Chang }{ }^{t} \text { nom } \\
\text { de genre }\end{array}$ \\
\hline Hinia incrassata (Ström) & Nassarius incrassatus (Ström) & Accord \\
\hline
\end{tabular}

Actualisation de la nomenclature d'après Appeltans et al. (2012)

\section{ANNEXE 2}

Actualisation de la liste de taxons présents dans le port du Havre, publiée par Breton (2005).

Taxons animaux, jusqu'en 2005.

\begin{tabular}{|c|c|}
\hline Liste de 2005 & Actualisation \\
\hline Bolinopsis infundibulum Müller & Mnemiopsis leidyi A. Agassiz \\
\hline Eupolymnia nebulosa (Montagu) & Terebellidae indét. \\
\hline Hemigrapsus penicillatus de Haan & Hemigrapsus takanoi Asakura \& Watanabe \\
\hline $\begin{array}{c}\text { Aplidium sp. 1 } \\
\text { Aplidium cf. califonicum Ritter } \\
\text { Aplidium densum (Giard) }\end{array}$ & Aplidium glabrum (Verrill) \\
\hline Didemnum cf. lahillei Hartmeyer & Didemnum vexillum Kott \\
\hline Gaidropsarus mediterraneus (Linnaeus) ? & Gadiformes indét. \\
\hline
\end{tabular}




\section{ANNEXE 3}

Liste systématique des taxons animaux nouvellement recensés dans le port du Havre postérieurement à l'article de Breton (2005), dressée par grands groupes informels puis par ordre alphabétique. Les espèces introduites sont en caractères gras.

\author{
Porifera \\ Dysidea fragilis Montagu \\ Mycale (Carmia) subclavata \\ Bowerbank \\ Pseudosuberites sulphureus Bean \\ in Bowerbank
}

\section{Cnidaria}

Clytia gracilis M. Sars ?

Hydrallmania falcata (Linneaus)

Obelia dichotoma (Linnaeus)

Tubularia larynx Ellis \& Solander

\section{Nemertea}

Lineus ruber (Müller)

Lineus sanguineus (Rathke)

Lineus viridis (Müller)

Tubulanus superbus (Kölliker)

\section{Annelida Polychaeta}

Amblyosyllis formosa (Claparède)

Bispira fabricii (Krøyer)

Bispira volutacornis (Montagu)

Flabelligera affinis M. Sars

Myxicola infundibulum (Renier)

Pseudopolydora pulchra (Corazzi)

Sthenelais boa (Johnston)

\author{
Abra tenuis (Montagu) \\ Acanthocardia paucicostata \\ (GB Sowerby II) \\ Arcopagia crassa (Pennant) \\ Chlamys varia (Linnaeus) \\ Crassostrea gigas (Thunberg) \\ Lutraria angustior Philippi \\ Ostrea edulis Linnaeus \\ Ruditapes philippinarum (Adams \\ \& Reeve)
}

Mollusca Gastropoda

Aeolidia papillosa (Linnaeus)

Elysia viridis (Montagu)

Hydrobia ulvae (Pennant)

Littorina mariae Sacchi \& Rastelli

Nucella lapillus (Linnaeus)

Tergipes tergipes Forsskål (pontes)

Trivia monacha (da Costa)

Hexapoda Apterygota

Petrobius maritimus (Leach)

Anurida maritima (Guérin-Méneville)

Crustacea Cirripedia

Semibalanus balanoides (Linnaeus)

Crustacea Isopoda

Idotea baltica (Pallas) 


\section{Crustacea Amphipoda}

Caprella linearis (Linnaeus)

Gammarus zaddachi Sexton

Talitrus saltator (Montagu)

Crustacea Decapoda

Ebalia tuberosa (Pennant)

Palaemon macrodactylus Rathbun

Bryozoa

Aetea sica (Couch)

Fenestrulina delicia Winston,

Hayward \& Craig

Membranipora membranacea (Linnaeus)

Ascidiacea

Aplidium punctum (Giard)

Ascidia conchilega O. F. Müller

Corella eumyota Traustedt
Lissoclinum perforatum (Giard) Ascidiella scabra (O. F. Müller)

Chondrichthyes

Scyliorhinus canicula (Linnaeus)

Osteichthyes

Entelurus aequoreus (Linnaeus) Eutrigla gurnardus (Linnaeus) Gobius couchi Miller \& El-Tawil Hippocampus hippocampus (Linnaeus) Raniceps raninus (Linnaeus) Sardinia pilchardus (Walbaum) Scophtalmus maximus (Linnaeus)

\section{Mammalia}

Phoca vitulina Linnaeus

Soient 60 taxons animaux, dont 5 espèces introduites $(8,3 \%)$

\section{ANNEXE 4}

Liste systématique de la faune du port d'Antifer (port pétrolier et port de service) du 01.04.2007 au 23.07.2011, dressée par grands groupes informels puis par ordre alphabétique. Les espèces introduites sont en caractères gras.

Porifera

Amphilectus fucorum (Esper) Aplysilla sulfurea? Schultze Dysidea fragilis Montagu Halichondria bowerbanki Burton Halichondria panicea (Pallas) Haliclona cinerea (Grant) Haliclona oculata (Pallas) Haliclona rosea (Bowerbank) Hymeniacidon perlevis (Montagu) Leuconia nivea Grant Leucosolenia variabilis (Haeckel) Microciona atrasanguinea Bowerbank
Mycale contarenii (Martens) Mycale macilenta (Bowerbank) Myxilla sp.

Oscarella lobularis (Schmidt)

Polymastia penicillus Montagu Prosuberites epiphytum (Lamarck) Suberites carnosus (Johnston) Suberites ficus (Esper)

Suberites massa Nardo Sycon ciliatum (Fabricius)

\section{Cnidaria}

Abietinaria abietina (Linnaeus) 
Actinia equina (Linnaeus)

Actinia fragacea Tugwell

Adamsia carciniopadosOtto

Alcyonium digitatum Linnaeus

Anemonia viridis Forsskål

Aurelia aurita (Linnaeus)

Cereus pedunculatus (Pennant)

Cerianthus Iloydi Gosse

Chrysaora hysoscella (Linnaeus)

Corymorpha nutans Sars

Cyanea lamarckii Péron \& Lesueur

Diphasia sp.

Halcampioides elongatus Carlgren in

Stephens

Hydractinia echinata (Fleming)

Kirchenpaueria sp.

Laomedea flexuosa Adler

Metridium senile (Linnaeus)

Obelia bidentata Clarcke

Obelia dichotoma (Linnaeus)

Obelia geniculata Linnaeus

Obelia longissima (Pallas)

Sagartia troglodytes (Price in Johnson)

Sertularella sp.

Tubularia indivisa Linnaeus

Tubularia larynx Ellis \& Solander

Urticina felina (Linnaeus)

\section{Ctenaria}

Mnemiopsis leidyi (A. Agassiz)

Pleurobrachia pileus (O. F. Müller)

Platyhelminthes

Prostheceraeus vittatus (Montagu)

Nemertea

Cerebratulus fuscus (Maclntosh)

Lineus longissimus (Gunnerus)

Tetrastemma sp.
Annelida Polychaeta

Aphrodita aculeata (Linnaeus) Arenicola marina (Linnaeus) Bispira volutacornis (Montagu) Chaetopterus variopedatus (Renier) Eulalia viridis (Linnaeus)

Eupolymnia nebulosa (Montagu)

Hydroides sp.

Lagis koreni (Malmgren)

Lanice conchilega (Pallas)

Myxicola infundibulum (Renier)

Polydora sp.

Sabella pavonina (Savigny)

Serpula vermicularis (Linnaeus)

Spirobranchus lamarcki (Quatrefages)

Spirobranchus triqueter (Linnaeus)

Spirorbis sp.

Mollusca Polyplacophora

Leptochitona cinereus (Linnaeus)

Acanthochitona crinita (Pennant)

Mollusca Bivalvia

Acanthocardia echinata (Linnaeus) Aequipecten opercularis (Linnaeus) Cerastoderma sp.

Chlamys varia (Linnaeus)

Ensis arcuatus (Jeffreys)

Ensis directus Conrad

Lutraria lutraria (Linnaeus)

Mytilus edulis Linnaeus

Ostrea edulis Linnaeus

Pecten maximus (Linnaeus)

Phaxas pellucidus Pennant

Polititapes virgineus

(Linnaeus) [=Venerupis rhomboides

(Pennant)]

Solen marginatus Pulteney

Spisula elliptica (Brown)

Venerupis corrugata (Gmelin) 
Mollusca Gastropoda

Aeolidia papillosa (Linnaeus)

Archidoris pseudoargus (Rapp)

Buccinum undatum Linnaeus

Crepidula fornicata (Linnaeus)

Epitonium clathrus (Linnaeus)

Euspira catena (da Costa)

Euspira pulchella (Risso) [= Polinices

poliana (delle Chiaje)]

Facelina bostoniensis (Couthouy)

Gibbula cineraria (Linnaeus)

Gibbula umbilicaris (Linnaeus)

Gibbula unbilicalis (da Costa)

Janolus cristatus (Delle Chiage)

Limacia clavigera (O. F. Müller)

Littorina littorea (Linnaeus)

Nassarius incrassatus (Ström)

Nassarius reticulatus (Linnaeus)

Nucella lapillus (Linnaeus)

Ocenebra erinaceus (Linnaeus)

Patella vulgata (Linnaeus)

Polycera faeroensis Lemche

Tergipes tergipes Forsskål (pontes)

Trivia arctica (Pulteney)

Trivia monacha (da Costa)

\section{Mollusca Cepahalopoda}

Alloteuthis subulatus (Lamarck)?

Loligo vulgaris (Lamarck)

Sepia officinalis Linnaeus

Sepiola atlantica d'Orbigny

\section{Crustacea Cirripedia}

Austrominius modestus (Darwin)

Semibalanus balanoides (Linnaeus)

Balanus crenatus Bruguière

Balanus improvisus Darwin

Verruca stroemia O. F. Müller

Megabalanus tintinnabulum (Linnaeus)

Crustacea Mysidacea
Praunus flexuosus (Müller)

Crustacea Isopoda

Idotea baltica (Pallas)

Crustacea Amphipoda

Phtisica marina Slabber

Crustacea Decapoda

Cancer pagurus Linnaeus

Carcinus maenas (Linnaeus)

Corystes cassivelaunus (Pennant)

Crangon crangon Linnaeus

Ebalia tuberosa (Pennant)

Galathea intermedia Liljeborg ?

Galathea squamifera Leach

Galathea strigosa Linnaeus

Hemigrapsus sanguineus de Haan

Hippolyte varians Leach

Homarus gammarus Linnaeus

Inachus phalangium Fabricius

Liocarcinus holsatus Fabricius

Macropodia rostrata (Linnaeus)

Necora puber(Linnaeus)

Nepinnotheres pinnotheres (Linnaeus)

[dans Ascidia mentula Müller]

Pagurus bernhardus (Linnaeus)

Pagurus cuanensis Thompson

Pagurus prideaux Leach

Palaemon serratus(Pennant)

Pilumnus hirtellus (Linnaeus)

Pisa tetraodon (Pennant)

Pisidia longicornis (Linnaeus)

Porcellana platycheles(Pennant)

Processidae indét.

Bryozoa

Aetea sp.

Alcyonidium gelatinosum (Linnaeus)

Amathia lendigera (Linnaeus) 
Bowerbankia citrina (Hincks)

Bugula flabellata (Thompson in Gray)

Bugula fulva Ryland

Bugula neritina (Linnaeus)

Bugula stolonifera Ryland

Bugula turbinata Alder

Conopeum reticulum (Linnaeus)

Conopeum seurati (Canu)

Cryptosula pallasiana (Moll)

Disporella hispida (Fleming)

Electra pilosa (Linnaeus)

Fenestrulina delicia

Winston, Hayward \& Craig

Flustra foliacea (Linnaeus)

Patinella verrucaria (Linnaeus)

[= Licheno-pora verrucaria

(O. Fabricius]

Membranipora membranacea

(Linnaeus)

Phaeostachys spinifera (Johnston)

Phoronida

Phoronis hippocrepia Wright

Echinodermata

Amphipholis squamata Delle Chiage Amphiura chiajei Forbes

Asterias rubens Linnaeus

Echinocardium cordatum (Pennant)

Ophiothrix fragilis Abildgaard

Ophiura ophiura (Linnaeus)

Psammechinus miliaris (Gmelin)

Thione sp.

Ascidiacea

Aplidium cf. nordmanni (Milne Edwards)

Aplidium punctum (Giard)

Ascidia mentula (O. F.Müller)

Ascidiella aspersa (O. F.Müller)

Ascidiella scabra (O. F.Müller)

Botrylloides violaceus Oka
Botryllus schlosseri (Pallas)

Ciona interstinalis (Linnaeus)

Clavelina lepadiformis (O. F.Müller)

Corella parallelogramma (O. F.Müller)

Diplosoma listerianum (Milne Edwards)

Distomus variolosus Gaertner

Microcosmus savignyi C. Monniot

Molgula socialis Alder

Perophora japonica Oka

Perophora listeri Wiegman

Polycarpa comata Alder

Styela clava Herdmann

Styela cf. rustica Linneaus [=Polycarpa

cf. rustica (Linnaeus)]

Chondrichthyes

Scyliorhinus canicula (Linnaeus)

Osteichthyes

Ammodytes tobianus Linnaeus Anguilla anguilla (Linnaeus) Apletodon dentatus (Facciolà) Atherina presbyter Cuvier Buglossidium luteum (Risso) Callionymus lyra Linnaeus? Clupeidae indét.

Conger conger (Linnaeus)

Ctenolabrus rupestris (Linnaeus)

Cyclopterus lumpus Linnaeus

Dicentrarchus labrax (Linnaeus)

Gadus morhua? Linnaeus

Gobius niger Linnaeus

Gobius paganellus Linnaeus

Gobiusculus flavescens (Fabricius)

Labrus bergylta Ascanius

Labrus mixtus Linnaeus [=Labrus bima-

culatus Linnaeus

Limanda limanda (Linnaeus)

Lipophrys pholis (Linnaeus)

Merlangius merlangus (Linnaeus)

Mullus surmuletus Linnaeus

Parablennius gattorugine (Linnaeus)

Pholis gunellus (Linnaeus) 
Platichtys flesus (Linnaeus)

Pleuronectes platessa Linnaeus

Pollachius pollachius (Linnaeus)

Pomatoschistus minutus (Pallas)

Pomatoschistus pictus (Malm)

Scophtalmus maximus (Linnaeus)

Solea solea (Linnaeus)

Symphodos melops (Linnaeus)

Syngnathus acus (Linnaeus)

Taurulus bubalis (Euphrasen)
Thorogobius ephippiatus (Lowe)

Trigla lucerna (Linnaeus)

Trisopterus luscus (Linnaeus)

Trisopterus minutus (Linnaeus)

Zeugopterus punctatus (Bloch)

Soient 237 taxons animaux, dont 13 espèces introduites $(5,5 \%)$

\section{ANNEXE 5}

Liste systématique de la flore et de la faune du port de Rouen (Bassin Saint-Gervais) du 16.10.2010 au 23.07.2012, dressée par grands groupes informels puis par ordre alphabétique. Les espèces introduites sont en caractères gras.

Cyanobacteria (Algues Bleues)

Merismopedia convoluta Bréb.

Oscillatoria anguina (Bory) Gomont

Oscillatoria curviceps Ag.

Oscillatoria proboscidea Gomont

Oscillatoria tenuis Agardh ex Gomont

Rhodophytes (Algues Rouges)

Compsopogon aeruginosus (J.

Agardh) Kütz.

Batrachospermum moliliforme Sirodot

Xanthophyta (Algues Jaunes)

Vaucheria spp.

Vaucheria compacta (Collins) Collins

Bacillariophyta (Diatomées)

cf. Melosira sp.

Chlorophyta (Algues vertes)

Cladophora fracta (O. F. Müller ex Vahl)

Kütz.

Cladophora glomerata (L.) Kütz.

Cladophora sp.

Mougeotia sp.

Oedogonium sp.
Zygnematales indét.

Bryophyta (Mousses et Hépatiques)

Bryale indét.

Fontinalis antipyretica Hedw.

Marchantia polymorpha L.

Magnoliophyta (Angiospermes)

Callitriche obtusangulata Le Gall

Callitriche sp.

Ceratophyllum demersum $\mathrm{L}$.

Ceratophyllum submersum L.

Elodea nuttalii (Planch.) H. St John

Glyceria fluitans (L.) R. Br.

Ludwigia palustris (L.) Elliott

Myriophyllum spicatum $\mathrm{L}$.

Myriophyllum verticillatum $\mathrm{L}$.

Nasturtium officinale $\mathrm{R}$. Br.

Oenanthe aquatica (L.) Poir. ?

Plantago lanceolata L.

Polygonum persicaria L.

Potamogeton lucens L. ?

Potamogeton pectinatus $\mathrm{L}$.

Potamogeton sp.

Ranunculus sp.

Rorippa amphibia (L.)

Sagittaria sagittifolia $\mathrm{L}$. forme

vallisnerifolia

Stellaria neglecta Weihe 
Vallisneria spiralis L.

Veronica anagallis-aquatica $\mathrm{L}$.

Porifera (Spongiaires)

Spongilla lacustris (Linnaeus) Ephydatia muelleri (Lieberkühn)

Platyhelminthes (Planaires)

Dendrocoelium lacteum Müller Dugesia sp.

Annelida Oligocheta

Oligocheta indét.

Annelida Hirudinea (Sangsues)

Erpobdella octooculata (Linnaeus)

Erpobdellidae indét. (Cocons, )

Glossosiphonia sp.

Haementaria costata (Müller)

Hemiclepsis marginata (O. F. Müller)

Piscicola geometra (Linnaeus)

Mollusca Bivalvia

Anodonta anatina (Linnaeus)

Corbicula fluminea (Rafinesque)

Dreissena polymorpha (Pallas)

Sphaerium corneum (Linnaeus)

Mollusca Gastropoda

Bithinia tentaculata (Linnaeus)

Menetus dilatatus (Gould)

Radix auricularia (Linnaeus)

Radix peregra (Müller)

Stagnicola sp.

Theodoxus fluviatilis (Linnaeus)

Viviparus fasciatus (Linnaeus)

Crustacea Isopoda

Asellus aquaticus (Linnaeus)

\section{Crustacea Amphipoda}

Chelicorophium curvispinum (G. O. Sars)

Dikerogammarus villosus (Sowinsky)

Echinogammarus berilloni (Catta)

Gammarus gr. pulex (Linnaeus)

Crustacea Decapoda

\section{Atyaephyra desmaresti (Millet)}

Orconectes limosus (Rafinesque)

Hexapoda (insectes)

Bereaidae ? (étuis)

Chironomidae (cf Chironomus sp.)

Coenagrion sp.

Ephemerella sp.

Eristalini

Melampophylax sp. ,

Sysira sp.

Bryozoa

Cristatella mucedo Cuvier

Fredericella sultana (Blumenbach)

Pectinatella magnifica (Leidy)

Plumatella repens (Linnaeus)

Agnathes

Petromyzon marinus Linnaeus

Osteichthyes (poissons osseux)

Abramis brama (Linnaeus)

Anguilla anguilla Linnaeus

Cottus gobio Linnaeus

Cyprinus carpio Linnaeus

Esox lucius Linnaeus

Mugilidae [Liza ramada (Risso)? ou

Chelon labrosus (Risso) ?]

Perca fluviatilis Linnaeus

Platichtys flesus (Linnaeus) juvéniles 
Liste complémentaire des poissons, issue de l'enquête auprès des pêcheurs (noms vernaculaires donnés par les pêcheurs)

Gymnocephalus cernuus (Linnaeus)?, Gremille ? (rapporté sous le nom imprécis de «Perchette »)

Ameiurus melas (Rafinesque),

Poisson-Chat

Rutilus rutilus (Linnaeus), Gardon
Salmo salar Linnaeus, Saumon

Salmo trutta fario Linnaeus, Truite

Salmo trutta trutta Linnaeus, Truite de mer

Scardinius erythrophtalmus (Linnaeus), Rotengle

Silurus glanis Linnaeus, Silure

Sander lucioperca (Linnaeus), Sandre

Soient 99 taxons, dont 14 espèces introduites (14\%) 